\title{
Consensus of Noisy Multiagent Systems with Markovian Switching Topologies and Time-Varying Delays
}

\author{
Yilun Shang \\ Department of Mathematics, Tongji University, Shanghai 200092, China \\ Correspondence should be addressed to Yilun Shang; shylmath@hotmail.com
}

Received 25 March 2015; Revised 26 May 2015; Accepted 21 July 2015

Academic Editor: Son Nguyen

Copyright ( 2015 Yilun Shang. This is an open access article distributed under the Creative Commons Attribution License, which permits unrestricted use, distribution, and reproduction in any medium, provided the original work is properly cited.

\begin{abstract}
Stochastic multiagent systems have attracted much attention during the past few decades. This paper concerns the continuous-time consensus of a network of agents under directed switching communication topologies governed by a time-homogeneous Markovian process. The agent dynamics are described by linear time-invariant systems, with random noises as well as time-varying delays. Two types of network-induced delays are considered, namely, delays affecting only the output of the agents' neighbors and delays affecting both the agents' own output and the output of their neighbors. We present necessary and sufficient consensus conditions for these two classes of multiagent systems, respectively. The design method of consensus gains allows for decoupling the design problem from the graph properties. Numerical simulations are implemented to test the effectiveness of our obtained results as well as the tightness of necessary/sufficient conditions.
\end{abstract}

\section{Introduction}

In the past few years, distributed coordination of multiagent systems has been considered by many researchers due to its broad applications in such areas as swarming of animals/ robots, cooperative unmanned aerial/underwater vehicles, distributed computation, air traffic control, and distributed sensor networks. One of the important issues in coordinated control is network based consensus protocol design. In this setting, consensus refers to every agent achieving agreement about some common or shared quantity by exchanging information according to a set of rules.

For the purpose of reaching consensus, important interaction details of agents in a system are mostly encoded by the communication graph of the system, which gives a general setting to study consensus and allows for the application of graph-theoretical notations and tools. Distributed computation over networks has been studied in the pioneering work of Tsitsiklis [1] and Chatterjee and Seneta [2] in systems and ergodicity theory. More recently, analytical frameworks for solving consensus problems were introduced by OlfatiSaber and Murray [3] and Jadbabaie et al. [4] based on graph and matrix theory. Since then numerous consensus protocols have been proposed, mostly for simple single- and doubleintegrator dynamics (see, e.g., [5-9] and references therein). It is pointed out that [10] design of consensus protocols for agent dynamics delineated, more generally, by linear timeinvariant systems is more challenging due to the possible existence of strictly unstable eigenvalues (poles) in the open-loop matrix. Necessary and sufficient consensus conditions for linear time-invariant systems were explored in [11-14] recently.

In many cases, the communication between agents is subject to stochastic perturbation - the connections change with time due to packet drops, agent failure, and various external disturbances. Therefore, the communication graphs underpinning the physical systems are better characterized by random switching networks. Stochastic consensus with singleand double-integrator dynamics has been well researched [15-21]. For example, asymptotic agreement of continuoustime single-integrator agent dynamics over Poisson random graphs is considered in [15]. The results are further extended in [16] to solve mean square consensus under directed and weighted independently switching random graphs. When the communication topology is described by a strictly stationary ergodic graph process, a necessary and sufficient condition for almost sure consensus of single-integrator agents is shown 
to be the connectivity of the mean topology with respect to a stationary distribution of the process [17]. For both discretetime and continuous-time multiagent systems with singleintegrator dynamics and balanced communication graphs, it has been shown in [21] that the ergodic Markov jump linear system achieves average consensus almost surely if and only if the union of topologies corresponding to the states of the Markov process is strongly connected. Similarly, for second-order discrete systems with (not necessarily ergodic) Markovian switching topologies, the necessary and sufficient condition for mean square consensus becomes that each union of graphs corresponding to the closed sets of positive recurrent states has a spanning tree [20]. Recently, this result is extended to linear time-invariant agent dynamics in [10] for both discrete- and continuous-time consensus.

It is well documented in the literature [22] that unmodeled delay effects in a feedback mechanism may destabilize an otherwise stable system. In multiagent systems, time-varying delays may arise naturally due to the asymmetry of interactions, the congestion of the communication channels, and the finite transmission speed. Moreover, noise/uncertainty frequently occurs to agents through, for example, communication errors and spurious measurements in communication systems. Therefore, it would be desirable to understand consensus problems in the setting of Markovian switching topologies with interactions affected by both time-varying delays and random noises.

In this paper, we investigate consensus problems for continuous-time multiagent systems with linear time-invariant agent dynamics under Markovian switching topologies, timevarying delays, and stochastic noises. In particular, we consider two types of communication delays: delays affecting both the state of the agents and that of their neighbors and delays affecting only the state of the agents' neighbors. A unified framework that considers these delays in continuous-time multiagent systems with fixed topology is first established in [23]. It is worth noting that although other interesting delay-dependent robustness results are reported in, for example, [24-26], the communication topologies considered are either static or switch deterministically.

This work deals with a group of identical agent dynamics, each of which follows a linear time-invariant system with white noises input. The information flow between agents is modeled by a time-homogenous Markov process, whose state space corresponds to all the possible communication patterns (directed graphs). We establish necessary and sufficient conditions to guarantee all agents asymptotically achieve an agreement in the mean square sense and in the almost sure sense for both types of time delays, respectively. When each graph corresponding to a state of the Markov process contains a spanning tree or is $d$-regular for a fixed $d \geq 1$, we show that the agents can reach consensus for suitable time-varying delays in terms of $M$-matrices if the agent dynamics is stabilizable. Conversely, if the consensus is achieved, the agent dynamics must be stabilizable and each union of graphs corresponding to the closed sets of positive recurrent states of the Markov process contains a spanning tree. The main mathematical techniques used here are based on the stability analysis of Markovian jump linear systems, stochastic differential delay equations, and graph and matrix theory.

The rest of the paper is organized as follows. Section 2 contains the problem formulation. Section 3 presents the main results. A couple of numerical examples are given in Section 4. The conclusion is drawn in Section 5.

Notation. Let $\mathbf{1}_{n}$ and $\mathbf{0}_{n}$ be the $n$-dimensional column vectors of all ones and all zeros, respectively. $I_{n}$ represents an $n \times n$ identity matrix. If the dimension is clear from the context, we sometimes suppress the subscript $n$. The sets of real and complex numbers are denoted by $\mathbb{R}$ and $\mathbb{C}$, respectively. The closed right half plane is signified by $\mathbb{C}^{+}$. We say $A>B$ ( $A \geq B$ ) if $A-B$ is positive definite (semidefinite), where $A$ and $B$ are symmetric matrices of the same dimensions. $A^{T}$ means the transpose of matrix $A$, while $A^{H}$ means its conjugate transpose. For a vector $x,\|x\|$ refers to its Euclidean norm; for a matrix $A,\|A\|=\sqrt{\operatorname{trace}\left(A^{T} A\right)}$ represents its trace norm. Let $\|\cdot\|_{\max }$ represent the max norm of a matrix, namely, the maximum of the absolute values of elements. For a matrix $A \in \mathbb{R}^{n \times n}$, its null space is designated by $\operatorname{Null}(A)=\{x \in$ $\left.\mathbb{R}^{n}: A x=\mathbf{0}\right\}$. By $A \otimes B$ we denote the Kronecker product of matrices $A$ and $B$, which admits the following properties: $(A \otimes B)(C \otimes D)=A C \otimes B D,(A \otimes B)^{-1}=A^{-1} \otimes B^{-1}$, and $(A \otimes B)^{T}=A^{T} \otimes B^{T}$. Denote by $\left\{\lambda_{i}(A)\right\}_{i=1}^{n}$ the eigenvalues of a matrix $A \in \mathbb{C}^{n \times n}$. Throughout this paper, we will order them in a nondecreasing order according to their modules: $\left|\lambda_{1}(A)\right| \leq\left|\lambda_{2}(A)\right| \leq \cdots \leq\left|\lambda_{n}(A)\right|$.

\section{Problem Formulation and Preliminary Results}

2.1. Graph and Consensus Properties. Let $\mathscr{G}=(\mathscr{V}, \mathscr{E}, \mathscr{A})$ represent a directed graph of order $N$, where $\mathscr{V}=\left\{v_{1}, v_{2}, \ldots, v_{N}\right\}$ is the set of nodes (agents) and $\mathscr{E} \subseteq \mathscr{V} \times \mathscr{V}$ is the set of directed edges. The ordered pair $\left(v_{i}, v_{j}\right) \in \mathscr{E}$ denotes a directed edge from node $v_{i}$ to node $v_{j}$, indicating that the information can be sent from agent $v_{i}$ to agent $v_{j}$. The weighted adjacency matrix $\mathscr{A}=\left(a_{i j}\right) \in \mathbb{R}^{N \times N}$ is defined by $a_{i j}>0$ if $\left(v_{j}, v_{i}\right) \in \mathscr{E}$ and $a_{i j}=0$ otherwise. Define the indegree matrix as a diagonal matrix $\mathscr{D}=\operatorname{diag}\left(d_{1}^{\text {in }}, \ldots, d_{N}^{\text {in }}\right)$, with $d_{i}^{\text {in }}=\sum_{j=1}^{N} a_{i j}$ being the in-degree of agent $v_{i}$. Similarly, the out-degree of agent $v_{i}$ is defined by $d_{i}^{\text {out }}=\sum_{j=1}^{N} a_{j i} . \mathscr{G}$ is said to be balanced if $d_{i}^{\text {in }}=d_{i}^{\text {out }}$ for all $i=1, \ldots, N$ [3]. Define the graph Laplacian matrix as $\mathscr{L}=\left(l_{i j}\right)=\mathscr{D}-\mathscr{A}$, which has all row sums equal to zero.

A sequence of edges $\left(v_{i_{1}}, v_{i_{2}}\right),\left(v_{i_{2}}, v_{i_{3}}\right), \ldots,\left(v_{i_{k-1}}, v_{i_{k}}\right)$, with $\left(v_{i_{j-1}}, v_{i_{j}}\right) \in \mathscr{E}$ for $j=2, \ldots, k$, is called a directed path from agent $v_{i_{1}}$ to agent $v_{i_{k}}$. We say that $\mathscr{G}$ contains a spanning tree if there is an agent (called root) such that every other agent can be connected by a directed path starting from the root. By Lemma 3.3 of [6], $\mathscr{G}$ contains a spanning tree if and only if $0=\lambda_{1}(\mathscr{L})<\left|\lambda_{2}(\mathscr{L})\right|$. Let $s$ be a positive integer. The union of $s$ graphs $\mathscr{G}_{1}=\left(\mathscr{V}, \mathscr{E}_{1}, \mathscr{A}_{1}\right), \ldots, \mathscr{G}_{s}=\left(\mathscr{V}, \mathscr{E}_{s}, \mathscr{A}_{s}\right)$ is denoted by $\bigcup_{i=1}^{s} \mathscr{G}_{i}=\left\{\mathscr{V}, \bigcup_{i=1}^{s} \mathscr{E}_{i}, \sum_{i=1}^{s} \mathscr{A}_{i}\right\}$.

For $t \geq 0$, let $\tau(t) \geq 0$ be a differentiable function which will stand for the time-varying communication delay. 
For $i=1, \ldots, N$, the dynamics of each agent $v_{i}$ in continuous time takes the following two different forms:

(i) with self-delay:

$$
\begin{aligned}
\dot{x}_{i}(t)= & A x_{i}(t-\tau(t))+B u_{i}^{w}(t)+\widehat{A} x_{i}(t-\tau(t)) \dot{w}_{i}(t) \\
& +\widehat{B} \widehat{u}_{i}^{w}(t),
\end{aligned}
$$

(ii) without self-delay:

$$
\dot{x}_{i}(t)=A x_{i}(t)+B u_{i}^{o}(t)+\widehat{A} x_{i}(t) \dot{w}_{i}(t)+\widehat{B} \widehat{u}_{i}^{o}(t),
$$

where $x_{i}(t) \in \mathbb{R}^{n}$ represents the state of agent $v_{i}$ at time $t$, $u_{i}^{w}(t), \widehat{u}_{i}^{w}(t), u_{i}^{o}(t), \widehat{u}_{i}^{o}(t) \in \mathbb{R}^{m}$ are control inputs of agent $v_{i}$ given by

$$
\begin{aligned}
& u_{i}^{w}(t)=K \sum_{j=1}^{N} a_{i j}(t)\left(x_{j}(t-\tau(t))-x_{i}(t-\tau(t))\right), \\
& \widehat{u}_{i}^{w}(t) \\
& =\widehat{K} \sum_{j=1}^{N} \sigma_{i j}(t) \dot{w}_{i j}(t)\left(x_{j}(t-\tau(t))-x_{i}(t-\tau(t))\right), \\
& u_{i}^{o}(t)=K \sum_{j=1}^{N} a_{i j}(t)\left(x_{j}(t-\tau(t))-x_{i}(t)\right), \\
& \widehat{u}_{i}^{o}(t)=\widehat{K} \sum_{j=1}^{N} \sigma_{i j}(t) \dot{w}_{i j}(t)\left(x_{j}(t-\tau(t))-x_{i}(t)\right),
\end{aligned}
$$

respectively, $A, \widehat{A} \in \mathbb{R}^{n \times n}, B, \widehat{B} \in \mathbb{R}^{n \times m}$ are system matrices, and $\left\{w_{i}(t), w_{i j}(t): i, j=1,2, \ldots, N\right\}$ are independent standard white noises. Here, $K, \widehat{K} \in \mathbb{R}^{m \times n}$ are common consensus gains to be designed later, and $\sigma_{i j}$ are referred to as the intensity of noise. To highlight the presence of noise, it is natural to define a noise graph $\widehat{\mathscr{G}}=(\mathscr{V}, \mathscr{E}, \widehat{\mathscr{A}})$ with the adjacency matrix $\widehat{\mathscr{A}}=\left(\sigma_{i j}\right) \in \mathbb{R}^{N \times N}$ satisfying $\sigma_{i j}>0$ if $\left(v_{j}, v_{i}\right) \in \mathscr{E}$ and $\sigma_{i j}=0$ otherwise. By definition, if viewing $\mathscr{G}$ and $\widehat{\mathscr{G}}$ as unweighted graphs, that is, the adjacency matrices are taken as binary ones, we have $\mathscr{G}=\widehat{\mathscr{G}}$. Likewise, the corresponding degree and Laplacian matrices are denoted by $\widehat{\mathscr{D}}=$ $\operatorname{diag}\left(\sigma_{1}, \ldots, \sigma_{N}\right)$ with $\sigma_{i}=\sum_{j=1}^{N} \sigma_{i j}$ and $\widehat{\mathscr{L}}=\left(\widehat{l}_{i j}\right)=\widehat{\mathscr{D}}-\widehat{\mathscr{A}}$, respectively.

Remark 1. The above dynamical models characterize system uncertainties with Gaussian white noise appearing as an exogenous input (similar treatment can be found in, e.g., [2730]). To see this, take $\widehat{A}=\zeta_{1} A$ and $\widehat{B}=\zeta_{2} B\left(\zeta_{1}, \zeta_{2}>0\right)$. System (1), for example, can be recast as

$$
\begin{aligned}
\dot{x}_{i}(t)= & A\left(1+\zeta_{1} \dot{w}_{i}(t)\right) x_{i}(t-\tau(t)) \\
+ & B \sum_{j=1}^{N}\left(K a_{i j}(t)+\widehat{K} \zeta_{2} \sigma_{i j}(t) \dot{w}_{i j}(t)\right) \\
& \cdot\left(x_{j}(t-\tau(t))-x_{i}(t-\tau(t))\right) .
\end{aligned}
$$

The perturbations are represented by a linear combination of gain matrices $K$ and $\widehat{K}$ to be determined. Moreover, if $\zeta_{1}=1$, we take $\widehat{K}=K$. (Although other choices are theoretically allowed as per Theorem 8 below, we make them equal in practice since one usually is not able to separate out the noise from the rest of the state.) Thus, the uncertainty reduces to the conventional form $a_{i j}(t)+\zeta_{2} \sigma_{i j}(t) \dot{w}_{i j}(t)$. We mention that other commonly studied uncertainties pertaining to the consensus problems include the measurement noises which only affect the received neighbors' states (e.g., [18]) and the additive plant noises (e.g., [31])

Multiagent system (2) with consensus protocols (5) and (6) considers only propagation delays for information transmitted from agent $v_{j}$ to agent $v_{i}$ on the communication network. Propagation delay has been addressed previously, for example, in works [24, 32-35]. Multiagent system (1) with consensus protocols (3) and (4) models both self-delay and neighboring delay. This scheme is relevant for dynamic agents with computation or reaction delays; see, for example, $[3,25,26,36,37]$. Although it would be more realistic to explore heterogeneous delays, we consider the uniform delay $\tau(t)$ as a first step, and this simplifies the derivation.

In the current work, we deal with delay robustness in both multiagent systems (1) and (2) over a stochastically timevarying interaction topology $\mathscr{G}(t)$ as well as its associated noise topology $\widehat{\mathscr{G}}(t)$, which is governed by a homogeneous continuous-time Markov process $\theta(t)$, taking value in the finite set $S=\{1,2, \ldots, s\}$. More precisely, $\mathscr{G}(t) \in\left\{\mathscr{G}_{1}, \ldots, \mathscr{G}_{s}\right\}$ and $\widehat{\mathscr{G}}(t) \in\left\{\widehat{\mathscr{G}}_{1}, \ldots, \widehat{\mathscr{G}}_{s}\right\} ; \mathscr{G}(t)=\mathscr{G}_{i}$ and $\widehat{\mathscr{G}}(t)=\widehat{\mathscr{G}}_{i}$ if and only if $\theta(t)=i$. For each $i$, the adjacency, degree, and Laplacian matrices for $\mathscr{G}_{i}\left(\widehat{\mathscr{G}}_{i}\right.$, resp.) will be denoted by $\mathscr{A}_{i}, \mathscr{D}_{i}$, and $\mathscr{L}_{i}$ $\left(\widehat{\mathscr{A}}_{i}, \widehat{\mathscr{D}}_{i}\right.$, and $\widehat{\mathscr{L}}_{i}$, resp.), respectively.

Definition 2. System (1) ((2), resp.) under control protocols (3) and (4) ((5) and (6), resp.) achieves consensus if there exist consensus gains $K, \widehat{K}$ such that, for any $x_{i}(0) \in \mathbb{R}^{n}$ and initial distribution of $\theta(0)$,

$$
\lim _{t \rightarrow \infty} \mathrm{E}\left(\left\|x_{i}(t)-x_{j}(t)\right\|^{2}\right)=0
$$

for any $i, j \in\{1,2, \ldots, N\}$.

We say matrix $A$ in (1) and (2) is Hurwitz (or stable) if every eigenvalue of $A$ has strictly negative real part; that is, it belongs to $\mathbb{C} \backslash \mathbb{C}^{+}$. The pair $(A, B)$ is called stabilizable if there exists $C \in \mathbb{R}^{m \times n}$ such that $A+B C$ is Hurwitz [38].

Assumption 3. The following assumptions are made throughout the paper:

(a) Communication graphs $\mathscr{G}_{1}, \mathscr{G}_{2}, \ldots, \mathscr{G}_{s}$ are balanced.

(b) Matrix $A$ is not Hurwitz.

(c) The white noises $w_{i}(t) \stackrel{\mathrm{d}}{=} w_{i j}(t)$ for all $i, j=1, \ldots, N$, where $\stackrel{\mathrm{d}}{=}$ means equality in distribution.

(d) The Markov process $\theta(t)$ is independent of the Brownian motions $w_{i}(t)(i=1, \ldots, N)$. 
Item (a) in Assumption 3 is also used in [10, 21]. Item (b) is meant to eliminate the triviality, since consensus can be reached by setting zero consensus gains if $A$ is Hurwitz. In this paper, we assume a special sort of noise-possibly due to the homogeneity of the communication channels between each agent and its neighbors - in which $w_{i j}(t)$ is independent of $j$ as item (c) indicated. The consensus in Definition 2 is defined in the sense of mean square convergence. This implies that the consensus can also be achieved in the almost sure sense in view of item (d) and the homogeneity of the Markov process (see Corollary 3.46 of $[39,40]$ ).

2.2. Exponential Stability for Delay Markovian Jump Systems. Denote by $(\Omega, \mathscr{F}, \mathscr{P})$ the underlying common probability space for the Markov process and Brownian motions discussed above. The homogeneous continuous-time Markov process $\theta(t)$ with generator $Q=\left(q_{i j}\right) \in \mathbb{R}^{s \times s}$ is formally given by

$$
\begin{aligned}
& \mathrm{P}(\theta(t+h)=j \mid \theta(t)=i) \\
& \quad= \begin{cases}q_{i j} h+o(h), & \text { if } i \neq j, \\
1+q_{i i} h+o(h), & \text { if } i=j,\end{cases}
\end{aligned}
$$

where $h>0$ and $h \rightarrow 0$. Here $q_{i j}$ is the transition rate from $i$ to $j$ if $i \neq j$, while $q_{i i}=-\sum_{j \neq i} q_{i j}$. As is known, the state space $S=\{1,2, \ldots, s\}$ of $\theta(t)$ can be decomposed uniquely into the form $S=\left\{J \cup S_{1} \cup \cdots \cup S_{r}\right\}$, where each $S_{j}(j=1, \ldots, r)$ is a closed communication class (i.e., closed set in the Markov process) of positive recurrent states and $J$ is a set of transient states [41].

Let $x(t) \in \mathbb{R}^{n}$ for $t \geq 0$. Consider a stochastic differential delay equation with Markovian switching of the form

$$
\begin{aligned}
d x(t)= & f(x(t), x(t-\tau(t)), \theta(t)) d t \\
& +g(x(t), x(t-\tau(t)), \theta(t)) d w(t),
\end{aligned}
$$

where $w(t)$ is an $m$-dimensional standard Brownian motion and $f: \mathbb{R}^{n} \times \mathbb{R}^{n} \times S \rightarrow \mathbb{R}^{n}$ and $g: \mathbb{R}^{n} \times \mathbb{R}^{n} \times S \rightarrow \mathbb{R}^{n \times m}$ are locally Lipschitz continuous satisfying the following.

Assumption 4. For each $i \in S$, there exist constants $\alpha_{i} \in \mathbb{R}$ and $\rho_{i}, \gamma_{i}, \beta_{i} \geq 0$ such that, for all $x, y \in \mathbb{R}^{n}$,
(a) $2 x^{T} f(x, 0, i) \leq \alpha_{i}\|x\|^{2}$;
(b) $\|f(x, 0, i)-f(x, y, i)\| \leq \rho_{i}\|y\|$;
(c) $\|g(x, y, i)\|^{2} \leq \gamma_{i}\|x\|^{2}+\beta_{i}\|y\|^{2}$.

Define $F=\operatorname{diag}\left(-\alpha_{1}-\rho_{1}-\gamma_{1}, \ldots,-\alpha_{s}-\rho_{s}-\gamma_{s}\right)-Q \in \mathbb{R}^{s \times s}$. A square matrix $F$ is called a nonsingular $M$-matrix if all the off-diagonal entries are nonpositive and $F^{-1}$ is a nonnegative matrix. A list of equivalent conditions for $M$-matrix can be found in [41]. The following result establishes the exponential stability of system (10).

Lemma 5 (see [42]). Assume that $F$ is a nonsingular $M$ matrix and $\left(\left(\rho_{1}+\beta_{1}\right)^{-1}, \ldots,\left(\rho_{s}+\beta_{s}\right)^{-1}\right)^{T}-F^{-1} \mathbf{1}_{s} \in \mathbb{R}^{s}$ is a positive vector. Then the trivial solution $x(t) \equiv 0$ of $(10)$ is exponentially stable in mean square if

$$
\dot{\tau}(t)<1-\kappa
$$

for all $t \geq 0$, where $\kappa=\max _{1 \leq i \leq s} b_{i}\left(\rho_{i}+\beta_{i}\right)$ and $\left(b_{1}, \ldots, b_{s}\right)^{T}=$ $F^{-1} \mathbf{1}$.

It is obvious that $\kappa<1$. Therefore, unbounded time delay is allowed. This is a desirable feature, for example, in delayed cellular neural networks, where delays are variable and in effect unbounded [43-46].

Remark 6. The existence of solutions to dynamical systems (1) and (2), in an even more general nonlinear setting, has been studied in [47]. Let $Z(t)=\left(x_{1}^{T}(t), \ldots, x_{N}^{T}(t)\right)^{T} \in \mathbb{R}^{n N}$. For $\tau>0$, let $\tau(t):[0, \infty) \rightarrow[0, \tau]$ be a continuous function. Denote by $C\left([-\tau, 0] ; \mathbb{R}^{n N}\right)$ the family of continuous functions from $[-\tau, 0]$ to $\mathbb{R}^{n N}$. It is shown that [47] for any bounded and $\mathscr{F}$-measurable initial condition $Z_{0} \in C\left([-\tau, 0] ; \mathbb{R}^{n N}\right)$ system (1) (as well as (2)) has a unique continuous solution $Z\left(t ; Z_{0}\right)$ on $t \geq-\tau$. Our strategy in the sequel is to first transfer the systems to error dynamics (see (13) and (26) below) and then address the stability of zero solution utilizing Lemma 5.

The objective of this paper is to reveal how stability analysis of differential delay equations, together with techniques used in matrix, Markov chain, and graph theory, can be applied to investigate stochastic consensus problems (1) and (2).

\section{Main Results}

In this section, we derive necessary and sufficient conditions for reaching consensus of noisy linear systems (1) and (2) under Markovian switching topologies and time-varying delays.

3.1. Consensus Conditions for Systems with Self-Delay. We first consider multiagent system (1) with protocols (3) and (4), where both self-delay and neighboring delay are factored in.

Let $\mathscr{L}(t)=\left(l_{i j}(t)\right)$ and $\widehat{\mathscr{L}}(t)=\left(\widehat{l}_{i j}(t)\right)$ signify the Laplacian matrices for the switching topologies $\mathscr{G}(t)$ and $\widehat{\mathscr{G}}(t)$ at time $t$. For $i=1, \ldots, N$, define the disagreement error for agent $v_{i}$ as $\delta_{i}(t)=x_{i}(t)-\bar{x}(t)$, where $\bar{x}(t)=(1 / N) \sum_{i=1}^{N} x_{i}(t)$ is the average state vector. Rearranging (1) with (3) and (4) gives

$$
\begin{aligned}
& \dot{\delta}_{i}(t)=A \delta_{i}(t-\tau(t))+B K \sum_{j=1}^{N} l_{i j}(t) \delta_{j}(t-\tau(t)) \\
& +\left(\widehat{A} \delta_{i}(t-\tau(t))+\widehat{B} \widehat{K} \sum_{j=1}^{N} \widehat{l}_{i j}(t) \delta_{j}(t-\tau(t))\right) \\
& \cdot \dot{w}_{i}(t),
\end{aligned}
$$

where we have used Assumption 3(a). Set $\delta(t)=\left(\delta_{1}^{T}(t), \ldots\right.$, $\left.\delta_{N}^{T}(t)\right)^{T} \in \mathbb{R}^{n N}$ and $w(t)=\left(w_{1}(t), \ldots, w_{N}(t)\right)^{T} \in \mathbb{R}^{N}$. 
Moreover, let $\delta^{\prime}(t)=\operatorname{diag}\left(\delta_{1}(t), \ldots, \delta_{N}(t)\right)$ be an $n N \times N$ block diagonal matrix. Consequently, (12) can be recast as

$d \delta(t)$

$$
\begin{aligned}
= & \left(I_{N} \otimes A-\mathscr{L}(t) \otimes B K\right) \delta(t-\tau(t)) d t \\
& +\left(I_{N} \otimes \widehat{A}-\widehat{\mathscr{L}}(t) \otimes \widehat{B} \widehat{K}\right) \delta^{\prime}(t-\tau(t)) d w(t) .
\end{aligned}
$$

Theorem 7 (necessary conditions). Suppose that Assumption 3 holds and system (1) with protocols (3) and (4) achieves consensus. Furthermore, assume that there exist $\xi_{i}>0$ for $i=1, \ldots, s$, and $\zeta_{1}, \zeta_{2}>0$ such that $\widehat{\mathscr{A}}_{i}=\xi_{i} \mathscr{A}_{i}, \widehat{A}=\zeta_{1} A$, and $\widehat{B}=\zeta_{2} B$. Then

(a) each union of the graphs $\mathscr{G}_{i}(i=1, \ldots, s)$ corresponding to all the states in the closed set $S_{j}$ for $j=1, \ldots, r$ has a spanning tree;

(b) $(A, B)$ is stabilizable.

Proof. To prove (a), let $\mathscr{G}_{S_{1}}=\bigcup_{i \in S_{1}} \mathscr{G}_{i}$ be the union graph corresponding to the closed set $S_{1}$. Without loss of generality, assume that $\mathscr{G}_{S_{1}}$ does not contain a spanning tree. Denote by $\mathscr{L}_{S_{1}}$ its Laplacian matrix. By Assumption 3(a), $\mathscr{L}_{S_{1}}^{T}$ is also a Laplacian matrix. It follows from Corollary 4.2 of [48] that there is a unit vector $c=\left(c_{1}, \ldots, c_{N}\right)^{T} \in \mathbb{R}^{N}$ such that $c^{T} \mathbf{1}=0$ and $\mathscr{L}_{S_{1}}^{T} c=\mathbf{0}$. Using Lemma 3.5 of [21], we obtain

$$
\operatorname{Null}\left(-\mathscr{L}_{S_{1}}^{T}\right)=\bigcap_{i \in S_{1}} \operatorname{Null}\left(-\mathscr{L}_{i}^{T}\right)
$$

Hence, $\mathscr{L}_{i}^{T} c=\mathbf{0}$ for any $i \in S_{1}$. There exists some $\Phi_{1}$ such that $\Phi=\left(1 / \sqrt{N}, c, \Phi_{1}\right) \in \mathbb{R}^{N \times N}$ is an orthogonal matrix.

Define $\widetilde{\delta}(t)=\left(\Phi^{-1} \otimes I_{n}\right) \delta(t)$. By partitioning $\widetilde{\delta}(t)$ in line with $\delta(t)$, that is, $\widetilde{\delta}(t)=\left(\widetilde{\delta}_{1}^{T}(t), \ldots, \widetilde{\delta}_{N}^{T}(t)\right)^{T}$ with each $\widetilde{\delta}_{i}(t) \epsilon$ $\mathbb{R}^{n}$, we get $\widetilde{\delta}_{1}(t) \equiv \mathbf{0}$ and $\widetilde{\delta}_{2}(t)=\sum_{i=1}^{N} c_{i} \delta_{i}(t)$ by definition. Moreover, by using (13) we have for $t \geq 0$

$$
\begin{aligned}
\dot{\tilde{\delta}}(t)= & \left(\Phi^{-1} \otimes A\right) \delta(t-\tau(t)) \\
& -\left(\Phi^{-1} \mathscr{L}(t) \otimes B K\right) \delta(t-\tau(t)) \\
& +\left(\Phi^{-1} \otimes \widehat{A}\right) \delta^{\prime}(t-\tau(t)) \dot{w}(t) \\
& -\left(\Phi^{-1} \widehat{\mathscr{L}}(t) \otimes \widehat{B} \widehat{K}\right) \delta^{\prime}(t-\tau(t)) \dot{w}(t) .
\end{aligned}
$$

If the initial value $\theta(0) \in S_{1}$, then $\mathscr{L}(t) \in\left\{\mathscr{L}_{i}\right\}_{i \in S_{1}}$ and $\widehat{\mathscr{L}}(t) \epsilon$ $\left\{\widehat{\mathscr{L}}_{i}\right\}_{i \in S_{1}}$. Using the assumption $\widehat{\mathscr{A}}_{i}=\xi_{i} \mathscr{A}_{i}$, we derive

$$
\dot{\tilde{\delta}}_{2}(t)=A \widetilde{\delta}_{2}(t-\tau(t))+\widehat{A} \sum_{i=1}^{N} c_{i} \delta_{i}(t-\tau(t)) \dot{w}_{i}(t)
$$

for $t \geq 0$.

If $A \neq 0$, take $\widetilde{\delta}_{2}(-\tau(0)) \notin \operatorname{Null}(A)$. Thus, $\lim _{t \rightarrow \infty} \mathrm{E}\left\|\widetilde{\delta}_{2}(t)\right\| \neq 0$ under Assumption 3(b). This contradicts with the consensus definition.
If $A=0$, we obtain $\dot{\mathrm{E}}_{2}(t)=\mathbf{0}$ and recall $\widetilde{\delta}_{2}(t)=$ $\sum_{i=1}^{N} c_{i} \delta_{i}(t)$. Since $c^{T} \mathbf{1}=0$, there exist two components $c_{i} \neq 0$ and $c_{j} \neq 0$ satisfying $c_{i} \neq c_{j}$. Take $\delta_{i}(0)=\mathbf{1}, \delta_{j}(0)=-\mathbf{1}$, and $\delta_{k}(0)=\mathbf{0}$ for all $k \in\{1, \ldots, N\} \backslash\{i, j\}$. It yields that $\lim _{t \rightarrow \infty} \mathrm{E}\left\|\widetilde{\delta}_{2}(t)\right\| \neq 0$. Again, it results in a contradiction.

To prove (b), assume that $(A, B)$ is not stabilizable. Then $A$ has an unstable and uncontrollable mode, denoted by $\lambda \in \mathbb{C}^{+}$. It follows from the Popov-Belevitch-Hautus controllability test [38] that there exists some complex vector $c \neq \mathbf{0}$ satisfying $c^{H} A=c^{H} \lambda$ and $c^{H} B=\mathbf{0}$.

Let $y(t)=\left(y_{1}(t), \ldots, y_{N}(t)\right)^{T}=\left(I_{N} \otimes c\right)^{H} \delta(t) \in \mathbb{R}^{N}$. By means of (13) and the assumptions that $\widehat{A}=\zeta_{1} A$ and $\widehat{B}=\zeta_{2} B$, we derive

$$
\begin{aligned}
\dot{y}(t) & \\
= & \left(I_{N} \otimes c^{H} A\right) \delta(t-\tau(t)) \\
& -\left(\mathscr{L}(t) \otimes c^{H} B K\right) \delta(t-\tau(t)) \\
& +\left(I_{N} \otimes c^{H} \widehat{A}\right) \delta^{\prime}(t-\tau(t)) \dot{w}(t) \\
& -\left(\widehat{\mathscr{L}}(t) \otimes c^{H} \widehat{B} \widehat{K}\right) \delta^{\prime}(t-\tau(t)) \dot{w}(t) \\
= & \lambda y(t-\tau(t)) \\
& +\lambda \zeta_{1} \operatorname{diag}\left(y_{1}(t-\tau(t)), \ldots, y_{N}(t-\tau(t))\right) \dot{w}(t) .
\end{aligned}
$$

Taking $y(-\tau(0)) \neq \mathbf{0}$, we see that $\liminf _{t \rightarrow \infty} \mathrm{E}\|y(t)\|>$ 0 since $\lambda$ is unstable. This contradicts with the consensus definition.

Note that the assumption $\widehat{\mathscr{A}}_{i}=\xi_{i} \mathscr{A}_{i}(i=1, \ldots, s)$ is only used in the proof of statement (a), while the assumptions $\widehat{A}=$ $\zeta_{1} A$ and $\widehat{B}=\zeta_{2} B$ are only used in the proof of statement (b).

If $(A, B)$ is stabilizable, there exists an $n \times n$ matrix $P>0$ such that

$$
P>A^{T} P A-A^{T} P B\left(B^{T} P B\right)^{-1} B^{T} P A
$$

by the Riccati inequality [49]. Similarly, if $(\widehat{A}, \widehat{B})$ is stabilizable, there exists an $n \times n$ matrix $\widehat{P}>0$ such that

$$
\widehat{P}>\widehat{A}^{T} \widehat{P} \widehat{A}-\widehat{A}^{T} \widehat{P} \widehat{B}\left(\widehat{B}^{T} \widehat{P} \widehat{B}\right)^{-1} \widehat{B}^{T} \widehat{P} \widehat{A} .
$$

Theorem 8 (sufficient conditions). Suppose that Assumption 3 holds, both $(A, B)$ and $(\widehat{A}, \widehat{B})$ are stabilizable, and $\mathscr{G}_{i}$ contains a spanning tree for every $i \in S$. If $F=-\rho I_{s}-Q$ is a nonsingular $M$-matrix and $(\rho+\beta)^{-1} \mathbf{1}_{s}-F^{-1} \mathbf{1}_{s}$ is a positive vector, then system (1) with protocols (3) and (4) achieves consensus for $\tau(t)$ satisfying

$$
\dot{\tau}(t)<1-(\rho+\beta) \cdot\left\|F^{-1} \mathbf{1}_{s}\right\|_{\max },
$$

where $\rho=\sqrt{\lambda_{n}(P) / \lambda_{1}(P)}, \beta=\lambda_{n}(\widehat{P}) / \lambda_{1}(\widehat{P})$, and $P$ and $\widehat{P}$ are given by (18) and (19), respectively.

Moreover, feasible consensus gains $K$ and $\widehat{K}$ are given by $K=\eta\left(B^{T} P B\right)^{-1} B^{T} P A$ with $\eta \geq 1 / \min _{2 \leq j \leq N, 1 \leq i \leq s}\left|\lambda_{j}\left(\mathscr{L}_{i}\right)\right|$ and $\widehat{K}=\widehat{\eta}\left(\widehat{B}^{T} \widehat{P} \widehat{B}\right)^{-1} \widehat{B}^{T} \widehat{P} \widehat{A}$ with $\widehat{\eta} \geq 1 / \min _{2 \leq j \leq N, 1 \leq i \leq s}\left|\lambda_{j}\left(\widehat{\mathscr{L}}_{i}\right)\right|$, respectively. 
Proof. The idea is to apply Lemma 5 to the error dynamics (13). It suffices to check Assumption 4 holds.

For item (a), $\alpha_{i}=0$ for all $i \in S$ clearly. To check item (b), we take a unitary matrix for each $i \in S$ : $\Phi_{i}=$ $\left(\mathbf{1}_{N} / \sqrt{N}, \phi_{i, 2}, \ldots, \phi_{i, N}\right)$, where $\phi_{i, j} \in \mathbb{C}^{N}$ satisfies $\mathscr{L}_{i} \phi_{i, j}=$ $\lambda_{j}\left(\mathscr{L}_{i}\right) \phi_{i, j}$ for $j=2, \ldots, N$. Given $i \in S$, by setting $\widetilde{\delta}=$ $\left(\Phi_{i}^{H} \otimes I_{n}\right) \delta$ (and partitioning it in conformity with that of $\delta$ as in Theorem 7), we derive that

$$
\begin{aligned}
\delta^{T} & \left(I_{N} \otimes A^{T}-\mathscr{L}_{i}^{T} \otimes K^{T} B^{T}\right)\left(I_{N} \otimes P\right) \\
\cdot & \left(I_{N} \otimes A-\mathscr{L}_{i} \otimes B K\right) \delta \\
& =\delta^{T}\left(I_{N} \otimes A^{T} P A-\mathscr{L}_{i}^{T} \mathscr{L}_{i} \otimes K^{T} B^{T} P B K\right) \delta \\
& =\widetilde{\delta}^{H}\left(I_{N} \otimes A^{T} P A-\Phi_{i}^{H} \mathscr{L}_{i}^{H} \mathscr{L}_{i} \Phi_{i} \otimes K^{T} B^{T} P B K\right) \tilde{\delta} \\
& =\sum_{j=2}^{N} \widetilde{\delta}_{j}^{H} \\
& \cdot\left(A^{T} P A-\left|\lambda_{j}\left(\mathscr{L}_{i}\right)\right|^{2} \eta^{2} A^{T} P B\left(B^{T} P B\right)^{-1} B^{T} P A\right) \widetilde{\delta}_{j} \\
\leq & \sum_{j=1}^{N} \widetilde{\delta}_{j}^{H} P \widetilde{\delta}_{j}=\delta^{T}\left(I_{N} \otimes P\right) \delta,
\end{aligned}
$$

where we have taken $K=\eta\left(B^{T} P B\right)^{-1} B^{T} P A$ with $\eta \geq$ $1 / \min _{2 \leq j \leq N, 1 \leq i \leq s}\left|\lambda_{j}\left(\mathscr{L}_{i}\right)\right|$ and used inequality (18). Note that $\eta$ is well defined, since $\mathscr{G}_{i}$ contains a spanning tree, and thus there is only one zero eigenvalue of $\mathscr{L}_{i}$ [48].

Since $P>0$, using $(21)$ we obtain

$$
\begin{aligned}
& \left\|\left(I_{N} \otimes A-\mathscr{L}_{i} \otimes B K\right) \delta\right\|^{2} \leq \frac{1}{\lambda_{1}(P)} \\
& \cdot \delta^{T}\left(I_{N} \otimes A^{T}-\mathscr{L}_{i}^{T} \otimes K^{T} B^{T}\right)\left(I_{N} \otimes P\right) \\
& \cdot\left(I_{N} \otimes A-\mathscr{L}_{i} \otimes B K\right) \delta \leq \frac{1}{\lambda_{1}(P)} \delta^{T}\left(I_{N} \otimes P\right) \delta \\
& \quad \leq \frac{\lambda_{n}(P)}{\lambda_{1}(P)}\|\delta\|^{2} .
\end{aligned}
$$

Hence, we take $\rho=\rho_{i}=\sqrt{\lambda_{n}(P) / \lambda_{1}(P)}$ independent of $i$.

To verify item $(\mathrm{c})$, note that

$$
\begin{aligned}
& \left\|\left(I_{N} \otimes \widehat{A}-\widehat{\mathscr{L}}_{i} \otimes \widehat{B} \widehat{K}\right) \delta^{\prime}\right\|^{2} \\
& =\operatorname{trace}\left(\delta^{\prime T}\left(I_{N} \otimes \widehat{A}^{T}-\widehat{\mathscr{L}}_{i}^{T} \otimes \widehat{K}^{T} \widehat{B}^{T}\right)\right. \\
& \left.\cdot\left(I_{N} \otimes \widehat{A}-\widehat{\mathscr{L}}_{i} \otimes \widehat{B} \widehat{K}\right) \delta^{\prime}\right)=\delta^{T}\left(I_{N} \otimes \widehat{A}^{T}-\widehat{\mathscr{L}}_{i}^{T}\right. \\
& \left.\otimes \widehat{K}^{T} \widehat{B}^{T}\right)\left(I_{N} \otimes \widehat{A}-\widehat{\mathscr{L}}_{i} \otimes \widehat{B} \widehat{K}\right) \delta \\
& =\left\|\left(I_{N} \otimes \widehat{A}-\widehat{\mathscr{L}}_{i} \otimes \widehat{B} \widehat{K}\right) \delta\right\|^{2} .
\end{aligned}
$$

Therefore, similarly as in the proof of (21) and (22) we obtain

$$
\left\|\left(I_{N} \otimes \widehat{A}-\widehat{\mathscr{L}}_{i} \otimes \widehat{B} \widehat{K}\right) \delta^{\prime}\right\|^{2} \leq \frac{\lambda_{n}(\widehat{P})}{\lambda_{1}(\widehat{P})}\|\delta\|^{2},
$$

where we have taken $\widehat{K}=\widehat{\eta}\left(\widehat{B}^{T} \widehat{P} \widehat{B}\right)^{-1} \widehat{B}^{T} \widehat{P} \widehat{A}$ with $\widehat{\eta} \geq$ $1 / \min _{2 \leq j \leq N, 1 \leq i \leq s}\left|\lambda_{j}\left(\widehat{\mathscr{L}}_{i}\right)\right|$. Since the fact that $\mathscr{G}_{i}$ contains a spanning tree implies that $\widehat{\mathscr{G}}_{i}$ also contains a spanning tree, $\widehat{\eta}$ is well defined with the same reason as above. Hence, we take $\gamma_{i}=0$ and $\beta=\beta_{i}=\lambda_{n}(\widehat{P}) / \lambda_{1}(\widehat{P})$ for all $i \in S$.

Remark 9. (a) The design of consensus gains $K$ and $\widehat{K}$ splits the design problem from the underlying communication topology. For example, $K$ is constructed based on the system matrices (18) and a multiplicative coefficient $\eta$ depending only on the graphs. Such a design procedure decouples the effects of agent dynamics and the network topologies, which simplifies the consensus design for the cases where the number of agents is large (see also $[10,13]$ ).

(b) When $n=m, A=0$, and $B=I_{n}$, we reproduce singleintegrator agent dynamics, and (18) and (19) always hold true. This can be viewed as a generalization of results in [21] by introducing random noise and time delay.

(c) The assumption in Theorem 8 about $F$ being a nonsingular $M$-matrix and $(\rho+\beta)^{-1} \mathbf{1}_{s}-F^{-1} \mathbf{1}_{s}$ having positive entries is easy to verify. Indeed, all the off-diagonal entries are nonpositive by the definition of generator $Q$. Thus, it suffices to show that $F^{-1}$ is nonnegative (this always holds if $n=1$ ) and ensure that every row sum of it is less than $(\rho+\beta)^{-1}$.

(d) There is a gap pertaining to graph connectivity between sufficient conditions (Theorem 8) and necessary conditions (Theorem 7). Comparing with the previous work [10] for noise-free and delay-free systems, we understand that the stronger connectivity requirement-each graph $\mathscr{G}_{i}$ contains a spanning tree-is introduced to accommodate the added noises and time-varying delays. Notice that the results are based on Lemma 5, which is about nonlinear systems. This also suggests the conditions derived here could be conservative. Notwithstanding, the study of weaker sufficient condition (e.g., using some algebraic methods) comparable to that of the necessary condition is an interesting future research.

3.2. Consensus Conditions for Systems without Self-Delay. Next, we study multiagent system (2) with protocols (5) and (6), where only neighboring delay is considered.

Similarly as above, for $i=1, \ldots, N$, define the disagreement error for agent $v_{i}$ as $\delta_{i}(t)=x_{i}(t)-\bar{x}(t)$, and $\bar{x}(t)=(1 / N) \sum_{i=1}^{N} x_{i}(t)$ is the average state vector. Under Assumption 3(a), (2) together with (5) and (6) yields

$$
\begin{aligned}
& \dot{\delta}_{i}(t)=A \delta_{i}(t)+B K \sum_{j=1}^{N} a_{i j}(t)\left(\delta_{j}(t-\tau(t))-\delta_{i}(t)\right) \\
& +\left(\widehat{A} \delta_{i}(t-\tau(t))\right. \\
& \left.+\widehat{B} \widehat{K} \sum_{j=1}^{N} \sigma_{i j}(t)\left(\delta_{j}(t-\tau(t))-\delta_{i}(t)\right)\right) \dot{w}_{i}(t) .
\end{aligned}
$$


Let $\delta(t)=\left(\delta_{1}^{T}(t), \ldots, \delta_{N}^{T}(t)\right)^{T} \in \mathbb{R}^{n N}, w(t)=\left(w_{1}(t), \ldots\right.$, $\left.w_{N}(t)\right)^{T} \in \mathbb{R}^{N}$, and $\delta^{\prime}(t)=\operatorname{diag}\left(\delta_{1}(t), \ldots, \delta_{N}(t)\right)$. We rewrite (25) in a compact form as

$$
\begin{aligned}
& d \delta(t)=\left(\left(I_{N} \otimes A-\mathscr{D}(t) \otimes B K\right) \delta(t)\right. \\
& +(\mathscr{A}(t) \otimes B K) \delta(t-\tau(t))) d t \\
& +\left(\left(I_{N} \otimes \widehat{A}-\widehat{\mathscr{D}}(t) \otimes \widehat{B} \widehat{K}\right) \delta^{\prime}(t)\right. \\
& \left.\quad+(\widehat{\mathscr{A}}(t) \otimes \widehat{B} \widehat{K}) \delta^{\prime}(t-\tau(t))\right) d w(t) .
\end{aligned}
$$

Theorem 10 (necessary conditions). Suppose that Assumption 3 holds and system (2) with protocols (5) and (6) achieves consensus for some constant delay $\tau$. Furthermore, assume that there exist $\xi_{i}>0$ for $i=1, \ldots, s$, and $\zeta_{1}, \zeta_{2}>0$ such that $\widehat{\mathscr{A}}_{i}=\xi_{i} \mathscr{A}_{i}, \widehat{A}=\zeta_{1} A$, and $\widehat{B}=\zeta_{2} B$. Then

(a) each union of the graphs $\mathscr{G}_{i}(i=1, \ldots, s)$ corresponding to all the states in the closed set $S_{j}$ for $j=1, \ldots, r$ has a spanning tree;

(b) $(A, B)$ is stabilizable.

Proof. Since consensus is achieved, we have $\lim _{t \rightarrow \infty} \| \delta(t)-$ $\delta(t-\tau) \|=0$ and $\lim _{t \rightarrow \infty}\left\|\delta^{\prime}(t)-\delta^{\prime}(t-\tau)\right\|=0$ for fixed $\tau$. Therefore, system (26) can be recast as

$$
\begin{aligned}
& d \delta(t)=\left(\left(I_{N} \otimes A-\mathscr{L}(t) \otimes B K\right) \delta(t-\tau)\right. \\
& \left.\quad+O(\|\delta(t)-\delta(t-\tau)\|) \cdot \mathbf{1}_{N n}\right) d t \\
& \quad+\left(\left(I_{N} \otimes \widehat{A}-\widehat{\mathscr{L}}(t) \otimes \widehat{B} \widehat{K}\right) \delta^{\prime}(t-\tau)\right. \\
& \left.\quad+O\left(\left\|\delta^{\prime}(t)-\delta^{\prime}(t-\tau)\right\|\right) \cdot \mathbf{1}_{N n}\right) d w(t) .
\end{aligned}
$$

Hence, Theorem 10 can be proved by the same reasoning as in Theorem 7.

As is noted below Theorem 7 , the assumption $\widehat{\mathscr{A}}_{i}=$ $\xi_{i} \mathscr{A}_{i}(i=1, \ldots, s)$ is only used in the proof of statement (a), while the assumptions $\widehat{A}=\zeta_{1} A$ and $\widehat{B}=\zeta_{2} B$ are only used in the proof of statement (b).

Recall that if $(A, B)$ is stabilizable, there exists a $C \in \mathbb{R}^{m \times n}$ such that $A+B C$ is Hurwitz. Therefore, by the Lyapunov stability theorem, there exists an $n \times n$ matrix $P>0$ such that

$$
(A+B C) P+P(A+B C)^{T}=-I .
$$

Define a symmetric matrix $R \in \mathbb{R}^{n \times n}$ by

$$
R=(A+B C)^{T} P+P(A+B C) .
$$

Theorem 11 (sufficient conditions). Suppose that Assumption 3 holds, both $(A, B)$ and $(\widehat{A}, \widehat{B})$ are stabilizable, and $\mathscr{G}_{i}$ is $d$-regular $(d \geq 1)$ for any $i \in S$. If $F=-(\alpha+\gamma) I_{s}-$ $\operatorname{diag}\left(\rho_{1}, \ldots, \rho_{s}\right)-Q$ is a nonsingular $M$-matrix and $\left(\left(\rho_{1}+\right.\right.$ $\left.\left.\beta_{1}\right)^{-1}, \ldots,\left(\rho_{s}+\beta_{s}\right)^{-1}\right)^{T}-F^{-1} \mathbf{1}_{s}$ is a positive vector, then system (2) with protocols (5) and (6) achieves consensus for $\tau(t)$ satisfying

$$
\dot{\tau}(t)<1-\max _{1 \leq i \leq s} b_{i}\left(\rho_{i}+\beta_{i}\right)
$$

where $\left(b_{1}, \ldots, b_{s}\right)^{T}=F^{-1} \mathbf{1}, \alpha=\left(\lambda_{n}(R)-1\right) /\left(2 \lambda_{1}(P)\right)$, $\gamma=2 \lambda_{n}(\widehat{P}) / \lambda_{1}(\widehat{P}), \rho_{i}=\sqrt{\lambda_{N n}\left(\mathscr{A}_{i}^{T} \mathscr{A}_{i} \otimes K^{T} B^{T} B K\right)}$, and $\beta_{i}=$ $2 \lambda_{N n}\left(\widehat{\mathscr{A}}_{i}^{T} \widehat{\mathscr{A}}_{i} \otimes \widehat{K}^{T} \widehat{B}^{T} \widehat{B} \widehat{K}\right)$. Here, $P, \widehat{P}$, and $R$ are given by (28), (19), and (29), respectively.

Moreover, feasible consensus gains $K$ and $\widehat{K}$ are given by $K=-C / d$ and $\widehat{K}=\widehat{\eta}\left(\widehat{B}^{T} \widehat{P} \widehat{B}\right)^{-1} \widehat{B}^{T} \widehat{P} \widehat{A}$ with $\widehat{\eta} \geq$ $1 / \min _{1 \leq i \leq s, 1 \leq j \leq N} \sigma_{j}^{i}$, respectively. Here, $\widehat{\mathscr{D}}_{i}=\operatorname{diag}\left(\sigma_{1}^{i}, \ldots, \sigma_{N}^{i}\right)$ for $i=1, \ldots, s$.

Proof. As in the proof of Theorem 8, we will apply Lemma 5 to the error dynamics (26).

To check item (a) in Assumption 4, we note that

$$
\begin{aligned}
2 \delta^{T} & \left(I_{N} \otimes A-\mathscr{D}_{i} \otimes B K\right) \delta=\delta^{T}\left(I_{N} \otimes A-\mathscr{D}_{i} \otimes B K\right. \\
& \left.+I_{N} \otimes A^{T}-\mathscr{D}_{i} \otimes K^{T} B^{T}\right) \delta \leq \lambda_{N n}\left(I_{N} \otimes A-\mathscr{D}_{i}\right. \\
& \left.\otimes B K+I_{N} \otimes A^{T}-\mathscr{D}_{i} \otimes K^{T} B^{T}\right)\|\delta\|^{2}=\lambda_{n}(A \\
& \left.-d B K+(A-d B K)^{T}\right)\|\delta\|^{2},
\end{aligned}
$$

where we have used the assumption that $\mathscr{G}_{i}$ is $d$-regular and the Rayleigh quotient inequality. Taking $K=-C / d$ in (31) and utilizing (28) and (29), we get

$$
\begin{aligned}
& 2 \delta^{T}\left(I_{N} \otimes A-\mathscr{D}_{i} \otimes B K\right) \delta \leq \lambda_{n}(A+B C \\
& \left.\quad+(A+B C)^{T}\right)\|\delta\|^{2}=\frac{1}{2 \lambda_{1}(P)} \\
& \quad \cdot \lambda_{n}\left(\left(A+B C+(A+B C)^{T}\right) \lambda_{1}(P) I\right. \\
& \left.\quad+\lambda_{1}(P) I\left(A+B C+(A+B C)^{T}\right)\right)\|\delta\|^{2} \leq \frac{1}{2 \lambda_{1}(P)} \\
& \quad \cdot \lambda_{n}\left(\left(A+B C+(A+B C)^{T}\right) P\right. \\
& \left.\quad+P\left(A+B C+(A+B C)^{T}\right)\right)\|\delta\|^{2}=\frac{1}{2 \lambda_{1}(P)} \lambda_{n}(R \\
& \quad-I)\|\delta\|^{2} .
\end{aligned}
$$

Therefore, we take $\alpha=\alpha_{i}=\left(\lambda_{n}(R)-1\right) /\left(2 \lambda_{1}(P)\right)$ for all $i \in S$.

For item (b), again by applying the Rayleigh quotient inequality we derive

$$
\begin{aligned}
\left\|\left(\mathscr{A}_{i} \otimes B K\right) \delta\right\|^{2} & =\delta^{T}\left(\mathscr{A}_{i}^{T} \otimes K^{T} B^{T}\right)\left(\mathscr{A}_{i} \otimes B K\right) \delta \\
& \leq \lambda_{N n}\left(\mathscr{A}_{i}^{T} \mathscr{A}_{i} \otimes K^{T} B^{T} B K\right)\|\delta\|^{2} \\
& =\frac{1}{d^{2}} \lambda_{N n}\left(\mathscr{A}_{i}^{T} \mathscr{A}_{i} \otimes C^{T} B^{T} B C\right)\|\delta\|^{2} .
\end{aligned}
$$

Therefore, we take $\rho_{i}=(1 / d) \sqrt{\lambda_{N n}\left(\mathscr{A}_{i}^{T} \mathscr{A}_{i} \otimes C^{T} B^{T} B C\right)}$ for $i \epsilon$ $S$.

To show (c), we recall the simple norm inequality $\| a+$ $b \|^{2} \leq 2\left(\|a\|^{2}+\|b\|^{2}\right)$. It suffices to find suitable $\gamma_{i}$ and $\beta_{i}$ so that the following two inequalities hold for $i \in S$ :

$$
\begin{aligned}
2\left\|\left(I_{N} \otimes \widehat{A}-\widehat{\mathscr{D}}_{i} \otimes \widehat{B} \widehat{K}\right) \delta^{\prime}\right\|^{2} & \leq \gamma_{i}\left\|\delta^{\prime}\right\|^{2}, \\
2\left\|\left(\widehat{\mathscr{A}}_{i} \otimes \widehat{B} \widehat{K}\right) \delta^{\prime}\right\|^{2} & \leq \beta_{i}\left\|\delta^{\prime}\right\|^{2} .
\end{aligned}
$$


Since $(\widehat{A}, \widehat{B})$ is stabilizable, we get $\widehat{P}>0$ such that (19) holds. Hence,

$$
\begin{aligned}
\delta^{T} & \left(I_{N} \otimes \widehat{A}^{T}-\widehat{\mathscr{D}}_{i}^{T} \otimes \widehat{K}^{T} \widehat{B}^{T}\right)\left(I_{N} \otimes \widehat{P}\right) \\
& \cdot\left(I_{N} \otimes \widehat{A}-\widehat{\mathscr{D}}_{i} \otimes \widehat{B} \widehat{K}\right) \delta \\
& =\delta^{T}\left(I_{N} \otimes \widehat{A}^{T} \widehat{P} \widehat{A}-\widehat{\mathscr{D}}_{i}^{T} \widehat{\mathscr{D}}_{i} \otimes \widehat{K}^{T} \widehat{B}^{T} \widehat{B} \widehat{K}\right) \delta=\sum_{j=1}^{N} \delta_{j}^{T} \\
& \cdot\left(\widehat{A}^{T} \widehat{P} \widehat{A}-\left(\sigma_{j}^{i}\right)^{2} \widehat{\eta}^{2} \widehat{A}^{T} \widehat{P} \widehat{B}\left(\widehat{B}^{T} \widehat{P} \widehat{B}\right)^{-1} \widehat{B}^{T} \widehat{P} \widehat{A}\right) \delta_{j} \\
& \leq \sum_{j=1}^{N} \delta_{j}^{T} \widehat{P} \delta_{j}=\delta^{T}\left(I_{N} \otimes \widehat{P}\right) \delta,
\end{aligned}
$$

where we have taken $\widehat{K}=\widehat{\eta}\left(\widehat{B}^{T} \widehat{P} \widehat{B}\right)^{-1} \widehat{B}^{T} \widehat{P} \widehat{A}$ with $\widehat{\eta} \geq$ $1 / \min _{1 \leq i \leq s, 1 \leq j \leq N} \sigma_{j}^{i}$. Here $\left\{\sigma_{j}^{i}\right\}$ are defined as in the statement of Theorem 11. Since $d \geq 1$, we have $\sigma_{j}^{i}>0$ for all $i$ and $j$. Therefore, $\hat{\eta}$ is well defined.

Note that

$$
\begin{aligned}
& \left\|\left(I_{N} \otimes \widehat{A}-\widehat{D}_{i} \otimes \widehat{B} \widehat{K}\right) \delta^{\prime}\right\|^{2} \\
& \quad=\operatorname{trace}\left(\delta^{\prime T}\left(I_{N} \otimes \widehat{A}^{T}-\widehat{\mathscr{D}}_{i}^{T} \otimes \widehat{K}^{T} \widehat{B}^{T}\right)\right. \\
& \left.\cdot\left(I_{N} \otimes \widehat{A}-\widehat{\mathscr{D}}_{i} \otimes \widehat{B} \widehat{K}\right) \delta^{\prime}\right)=\delta^{T}\left(I_{N} \otimes \widehat{A}^{T}-\widehat{\mathscr{D}}_{i}^{T}\right. \\
& \left.\quad \otimes \widehat{K}^{T} \widehat{B}^{T}\right)\left(I_{N} \otimes \widehat{A}-\widehat{\mathscr{D}}_{i} \otimes \widehat{B} \widehat{K}\right) \delta \\
& =\left\|\left(I_{N} \otimes \widehat{A}-\widehat{\mathscr{D}}_{i} \otimes \widehat{B} \widehat{K}\right) \delta\right\|^{2} .
\end{aligned}
$$

Using (36) we obtain

$$
\begin{aligned}
& \left\|\left(I_{N} \otimes \widehat{A}-\widehat{\mathscr{D}}_{i} \otimes \widehat{B} \widehat{K}\right) \delta^{\prime}\right\|^{2} \leq \frac{1}{\lambda_{1}(\widehat{P})} \\
& \cdot \delta^{T}\left(I_{N} \otimes \widehat{A}^{T}-\widehat{\mathscr{D}}_{i}^{T} \otimes \widehat{K}^{T} \widehat{B}^{T}\right)\left(I_{N} \otimes \widehat{P}\right) \\
& \cdot\left(I_{N} \otimes \widehat{A}-\widehat{D}_{i} \otimes \widehat{B} \widehat{K}\right) \delta \leq \frac{1}{\lambda_{1}(\widehat{P})} \delta^{T}\left(I_{N} \otimes \widehat{P}\right) \delta \\
& \quad \leq \frac{\lambda_{n}(\widehat{P})}{\lambda_{1}(\widehat{P})} \delta^{T} \delta \leq \frac{\lambda_{n}(\widehat{P})}{\lambda_{1}(\widehat{P})}\left\|\delta^{\prime}\right\|^{2} .
\end{aligned}
$$

Therefore, (34) holds by taking $\gamma=\gamma_{i}=2 \lambda_{n}(\widehat{P}) / \lambda_{1}(\widehat{P})$ for any $i \in S$.

Similarly, (35) is true with $\beta_{i}=2 \lambda_{N n}\left(\widehat{\mathscr{A}}_{i}^{T} \widehat{\mathscr{A}}_{i} \otimes \widehat{K}^{T} \widehat{B}^{T} \widehat{B} \widehat{K}\right)$ by using the Rayleigh quotient inequality.

Remark 12. (a) Similar comments in Remark 9 can be applied here. In addition, we note that the requirement in Theorem $11-\mathscr{G}_{i}$ are $d$-regular graphs for all $i-$ is somewhat restrictive. If this condition is violated, we might use Weyl's inequality (see, e.g., [50]) to bound the maximum eigenvalue in (31), which nonetheless will lead to a more cumbersome expression.

(b) Interestingly, sufficient conditions in Theorem 11 do not explicitly mention connectivity assumptions, whereas
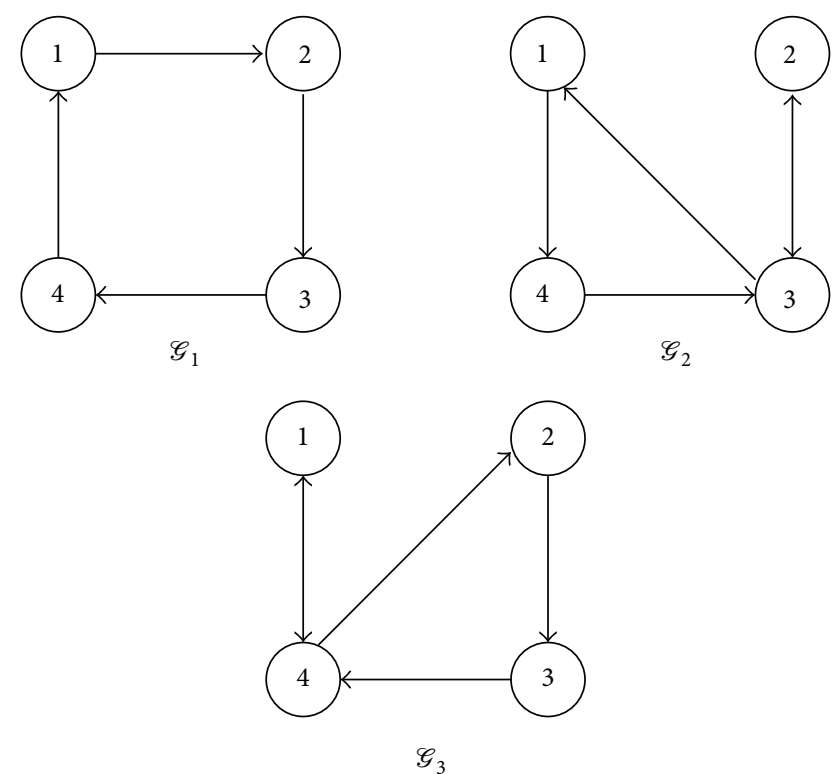

FigURE 1: Communication topologies $\mathscr{G}_{1}, \mathscr{G}_{2}$, and $\mathscr{G}_{3}$ for system (1).

necessary conditions in Theorem 10 clearly state certain connectivity assumption. To see how some connectivity is implicitly required in Theorem 11, we consider a special case with $n=m=1, S=\{1,2\}$, and $\mathscr{G}_{i}(i \in S)$ being not connected and having two connected components, out of which one is a complete graph with $d+1$ nodes. We can show that matrix $F$ in Theorem 11 is not a nonsingular $M$-matrix, violating the assumption of Theorem 11. Indeed, it is straightforward to check that $\gamma=2, \rho_{1}=\rho_{2}=d|B K|$, and $\alpha=2(A+B C)<0$ with $A \geq 0$. Setting $Q=\left(\begin{array}{cc}-a & a \\ b & -b\end{array}\right)$ with $a, b>0$, we obtain the $2 \times 2$ matrix $F$. $F$ being a nonsingular $M$-matrix is equivalent to the fact that all its leading principal minors are positive [41], which in turn yields $-2(A+B C)-2-d|B K|>0$. However, this inequality does not hold for any $K \in \mathbb{R}$ when $A+B C \geq-1$.

\section{Simulation Examples}

In this section, we present several examples to illustrate the availability of the proposed results. We consider all the adjacency matrices as binary $0-1$ matrices in the following.

Example 1 (system with self-delay). Consider multiagent system (1) with $N=4$ agents. The communication topologies will randomly switch amongst the triad $\mathscr{G}_{1}, \mathscr{G}_{2}$, and $\mathscr{G}_{3}$ of Figure 1 following a homogeneous Markovian process with generator

$$
Q=\left(\begin{array}{ccc}
-2 & 1 & 1 \\
1 & -4 & 3 \\
1 & 1 & -2
\end{array}\right)
$$

and state space $S=\{1,2,3\}$. Note that each of these three graphs is balanced and contains a spanning tree. For 


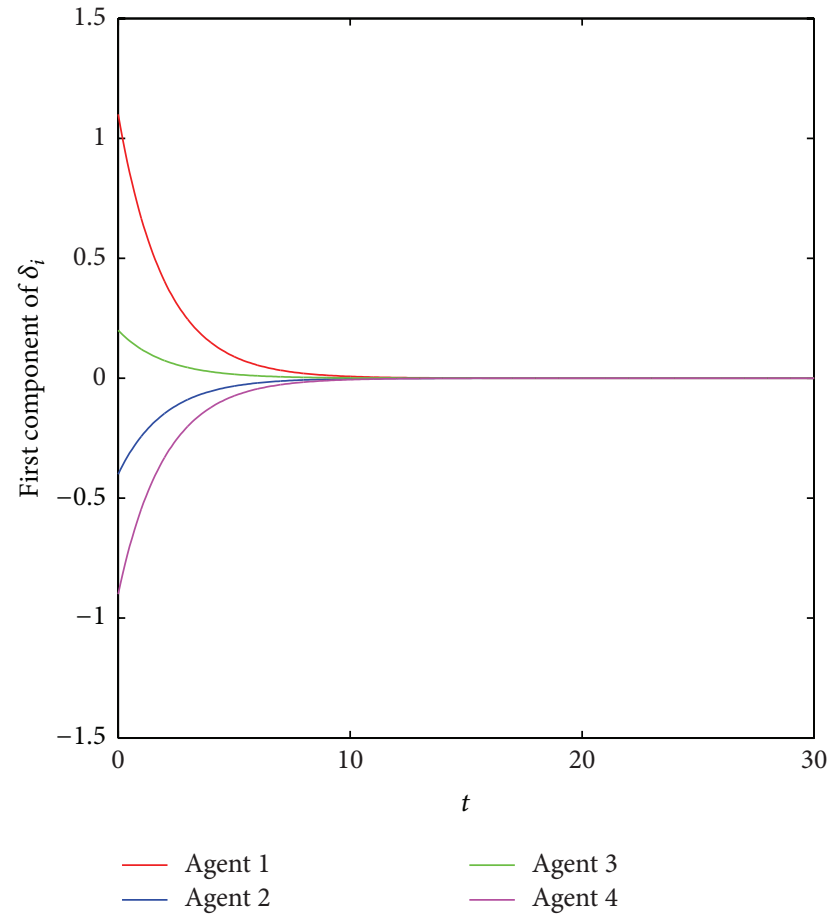

(a) $\tau(t)=0.05 \sin ^{2}(t)$

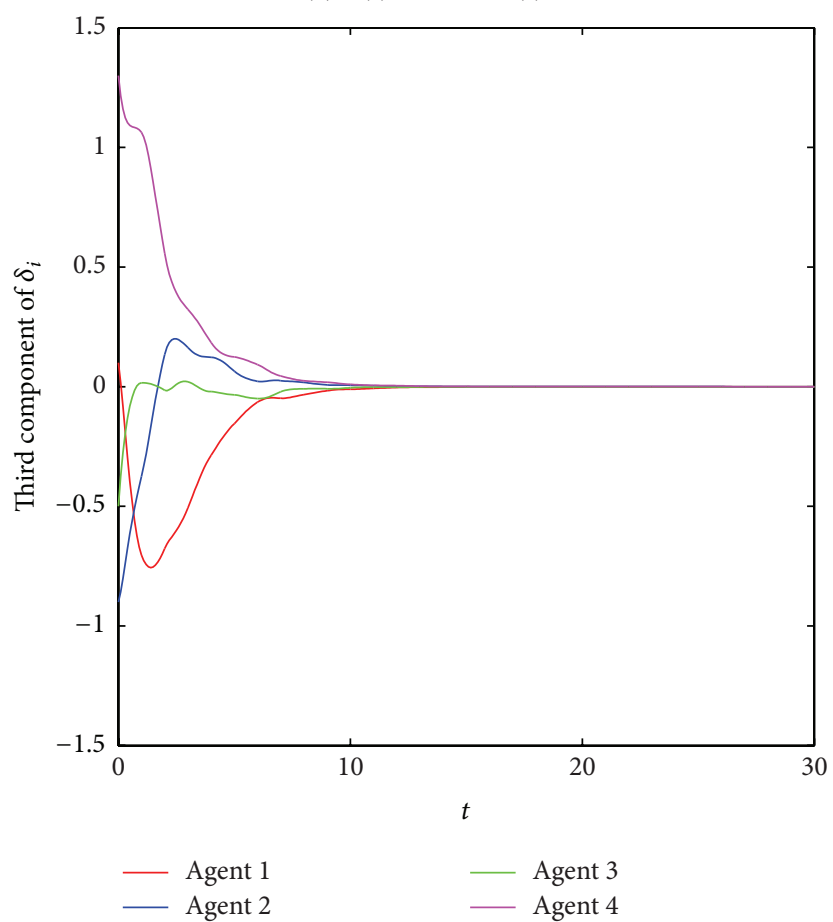

(c) $\tau(t)=0.05 \sin ^{2}(t)$

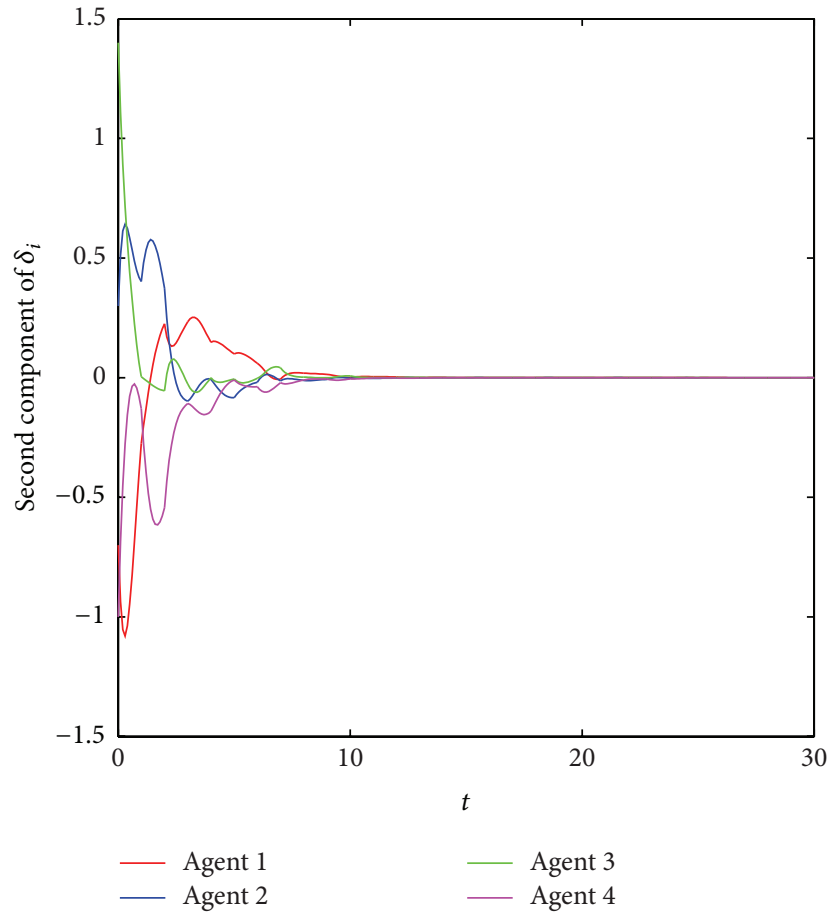

(b) $\tau(t)=0.05 \sin ^{2}(t)$

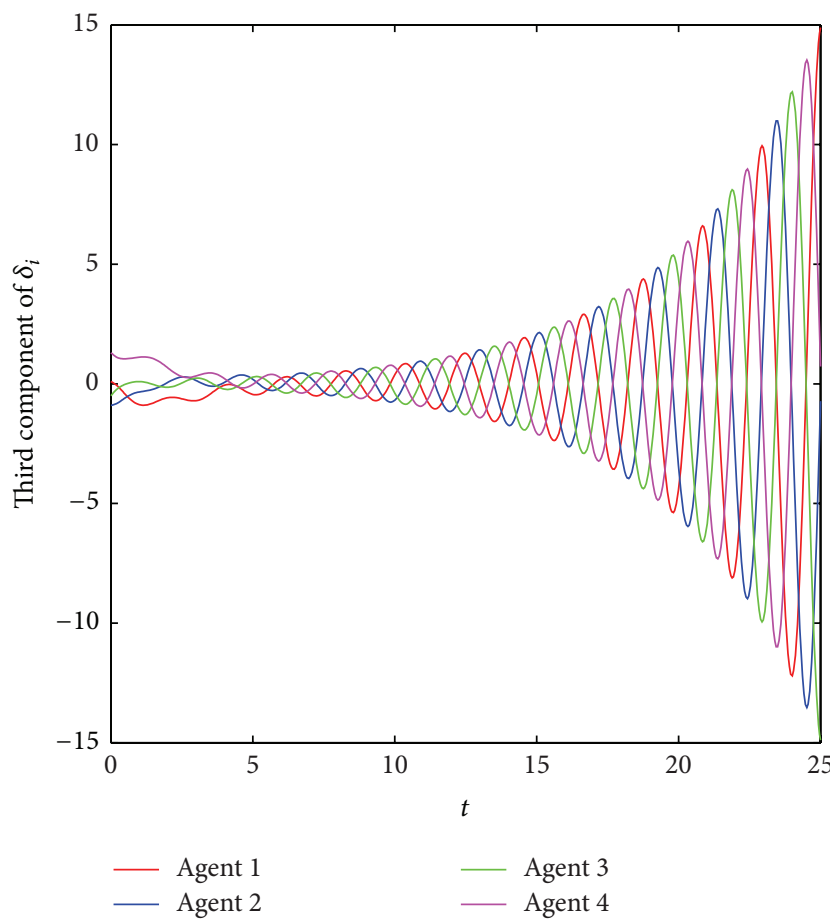

(d) $\tau(t)=0.2 \sin ^{2}(t)$

Figure 2: Consensus errors of multiagent system (1) with respect to different time-delay $\tau(t)$.

$i=1,2,3$, we choose $\widehat{\mathscr{A}}_{i}=0.01 \cdot \mathscr{A}_{i}$. Take $n=3, m=1$ and let the agent dynamics be specified as

$$
A=\left(\begin{array}{ccc}
-0.5 & 0 & 0 \\
0 & 1 & 0 \\
0 & 1 & 0
\end{array}\right) \text {, }
$$

$$
B=\widehat{B}=\left(\begin{array}{l}
0 \\
1 \\
0
\end{array}\right),
$$

and $\widehat{A}=0.01 \cdot A$. Since $A+B C$ and $\widehat{A}+\widehat{B} \widehat{C}$ are Hurwitz by choosing $C=(-1,-2,-1)$ and $\widehat{C}=0.01 \cdot C$, both pairs $(A, B)$ 
and $(\widehat{A}, \widehat{B})$ are stabilizable. Since Riccati inequalities (18) and (19) can be written as linear matrix inequalities (LMIs) (see, e.g., [51]), we obtain $P=\widehat{P}=\operatorname{diag}(1,1.1,1)$ by using LMI Toolbox in MATLAB. We calculate

$$
F^{-1}=\left(\begin{array}{lll}
0.1807 & 0.1295 & 0.0904 \\
0.3168 & 0.0565 & 0.0132 \\
0.0168 & 0.2832 & 0.0681
\end{array}\right)
$$

which is a nonnegative matrix. In view of Theorem 8 , we solve the consensus gains as $K=\widehat{K}=(2,2.5,2)$.

According to Theorem 8, multiagent system (1) with protocols (3) and (4) achieves consensus for $\tau(t)$ satisfying

$$
\begin{aligned}
\dot{\tau}(t) & <1-(\rho+\beta) \cdot\left\|F^{-1} \mathbf{1}_{s}\right\|_{\max } \\
& =1-(\sqrt{1.1}+1.1) \times 0.4006=0.1392 .
\end{aligned}
$$

With the initial state of each agent being taken randomly from $[-1,1]$, we show in Figures 2(a)-2(c) a sample path of the consensus errors $\delta_{i}(i=1, \ldots, 4)$ by choosing $\tau(t)=0.05 \sin ^{2}(t)$. Clearly, (42) is satisfied, and consensus is achieved. In Figure 2(d), we show only the third component of $\delta_{i}(i=1, \ldots, 4)$ with $\tau(t)=0.2 \sin ^{2}(t)$. This delay violates condition (42), and consensus is not achieved.

Example 2 (system without self-delay). Consider multiagent system (2) with $N=4$ agents. The communication topologies switch amongst $\mathscr{G}_{1}, \mathscr{G}_{2}$, and $\mathscr{G}_{3}$ of Figure 3 following the same Markovian process as defined in Example 1. Note that each of these three graphs is balanced and $d$-regular with $d=1 . \mathscr{G}_{1}$ and $\mathscr{G}_{3}$ contain spanning trees but $\mathscr{G}_{2}$ does not. The adjacency matrices $\mathscr{A}_{i}, \widehat{\mathscr{A}}_{i}(i=1,2,3)$ and the agent dynamics $A, B, \widehat{A}, \widehat{B}$ are taken as in Example 1. Solving Lyapunov equation (28) by using LYAP function in MATLAB, we obtain

$$
P=\left(\begin{array}{ccc}
1 & -0.2857 & -0.5714 \\
-0.2857 & 1.2857 & -0.5000 \\
-0.5714 & -0.5000 & 2.3571
\end{array}\right) \text {, }
$$

and $\widehat{P}=\operatorname{diag}(1,1.1,1)$ as in Example 1. It follows from (29) that

$$
R=\left(\begin{array}{ccc}
-1 & 0.2857 & 0 \\
0.2857 & -2.5714 & 0 \\
0 & 0 & 0
\end{array}\right)
$$

We calculate $\alpha=-0.9690, \gamma=2.2$, and a nonnegative matrix

$$
F^{-1}=\left(\begin{array}{lll}
0.2307 & 0.1453 & 0.0786 \\
0.1722 & 0.1204 & 0.2548 \\
0.0470 & 0.3793 & 0.0682
\end{array}\right) \text {. }
$$

From Theorem 11, we solve the consensus gains as $K=\widehat{K}=$ $(1,2,1)$.
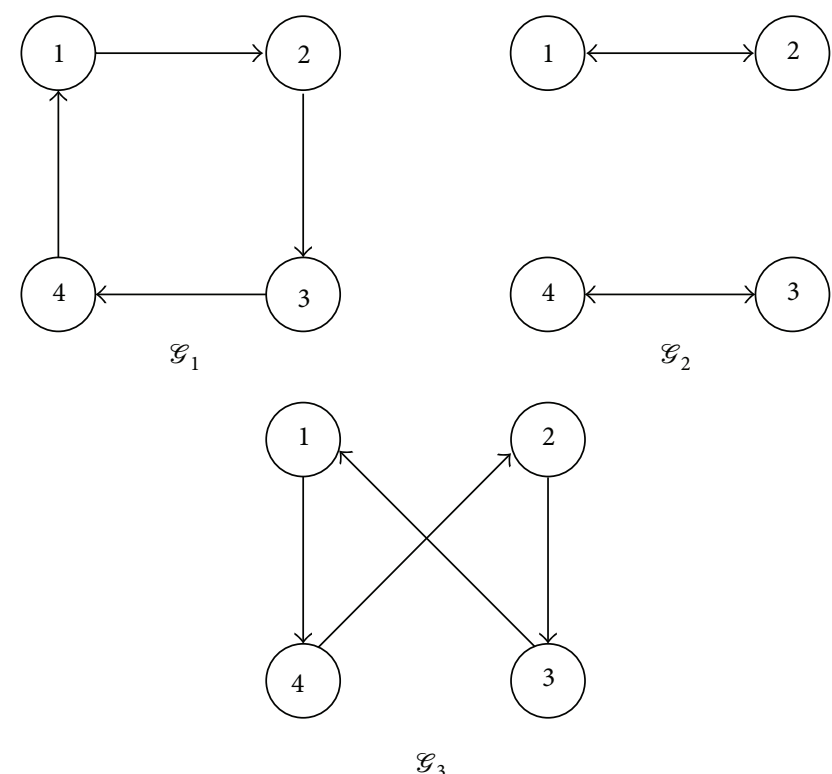

FIgURE 3: Communication topologies $\mathscr{G}_{1}, \mathscr{G}_{2}$, and $\mathscr{G}_{3}$ for system (2).

In light of Theorem 11, multiagent system (2) with protocols (5) and (6) achieves consensus for $\tau(t)$ satisfying

$$
\begin{aligned}
\dot{\tau}(t) & <1-\max _{1 \leq i \leq 3} b_{i}\left(\rho_{i}+\beta_{i}\right) \\
& =1-\max \{0.2510,0.7557,0.0586\}=0.2443
\end{aligned}
$$

With the initial state of each agent being taken randomly from $[-1,1]$, we show in Figures $4(\mathrm{a})-4(\mathrm{c})$ a sample path of the consensus errors $\delta_{i}(i=1, \ldots, 4)$ by choosing $\tau(t)=$ $0.1 \cos ^{2}(t)$. Since (46) is satisfied, consensus is achieved. We plot in Figure 4(d) only the third component of $\delta_{i}(i=$ $1, \ldots, 4)$ with $\tau(t)=0.3 \cos ^{2}(t)$. Condition (46) does not hold, and consensus is not achieved.

Example 3 (tightness of bounds). In this example, we consider multiagent system (1) with $N=1000$ agents. The communication topologies will randomly switch amongst 10 random graphs $\mathscr{G}_{1}, \ldots, \mathscr{G}_{10}$ in the classical Erdős-Rényi $\mathscr{G}(N, p)$ model with $p$ being the connection probability between any two nodes [52]. The generator of the underlying Markovian process $\theta(t)$ with $S=\{1,2, \ldots, 10\}$ is taken as $Q=-10 I_{10}+\mathbf{1}_{10} \mathbf{1}_{10}^{T}$. The adjacency matrices $\mathscr{A}_{i}, \widehat{\mathscr{A}}_{i}(i=$ $1,2, \ldots, 10)$ and the agent dynamics $A, B, \widehat{A}, \widehat{B}$ are taken as in Example 1. Moreover, we fix $\tau(t) \equiv 0.05$.

The program stops if all components of $\delta_{i}(i=1,2, \ldots$, 10) are less than $10^{-5}$; if the program does not stop before $t=10^{4}$, we regard that the consensus is not achieved. For each given $p$, we collect 500 samples (1 sample consists of 10 graphs) to check whether the system finally achieves consensus. The fraction of samples that reach consensus is shown in Figure 5 as a function of $p$. The curve displays a sigmoidal variation with respect to $p$, saturating at 1 when $p$ is just over $3.1 \times 10^{-3}$. It is well known that [52] the random graphs are not connected with high probability if 


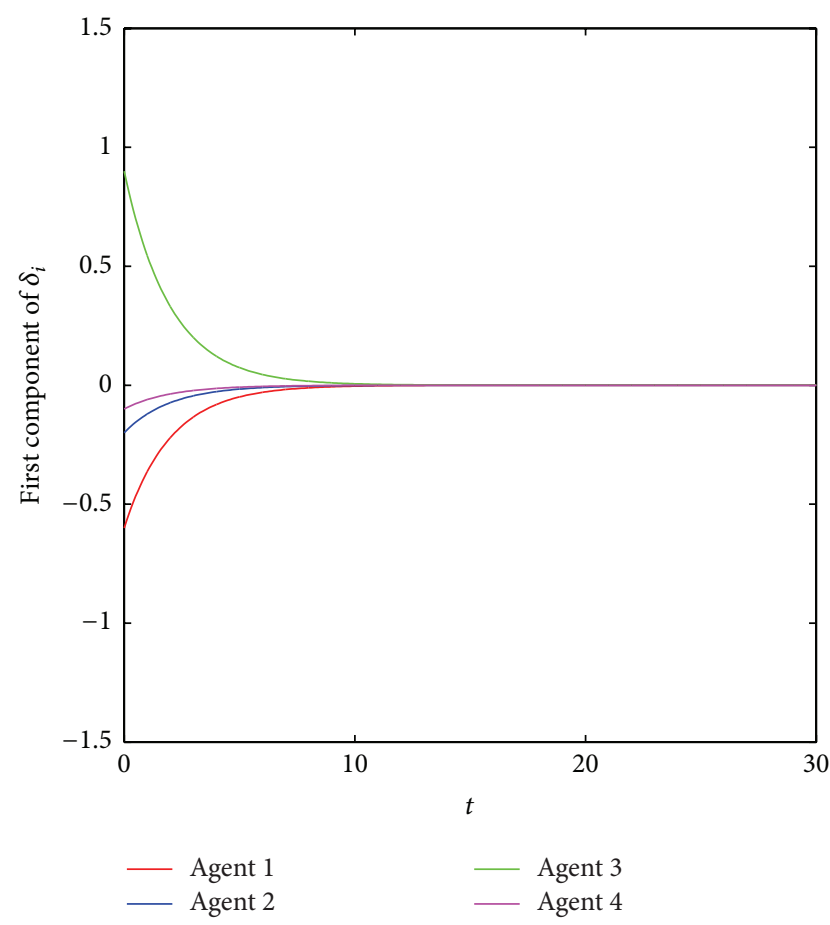

(a) $\tau(t)=0.1 \cos ^{2}(t)$

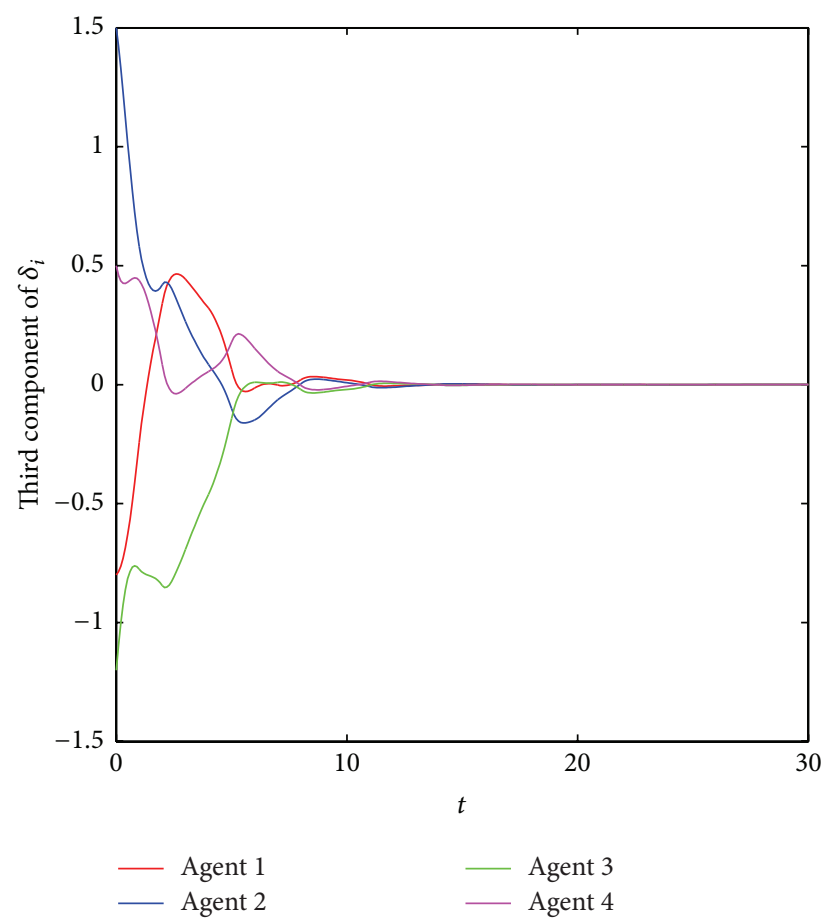

(c) $\tau(t)=0.1 \cos ^{2}(t)$

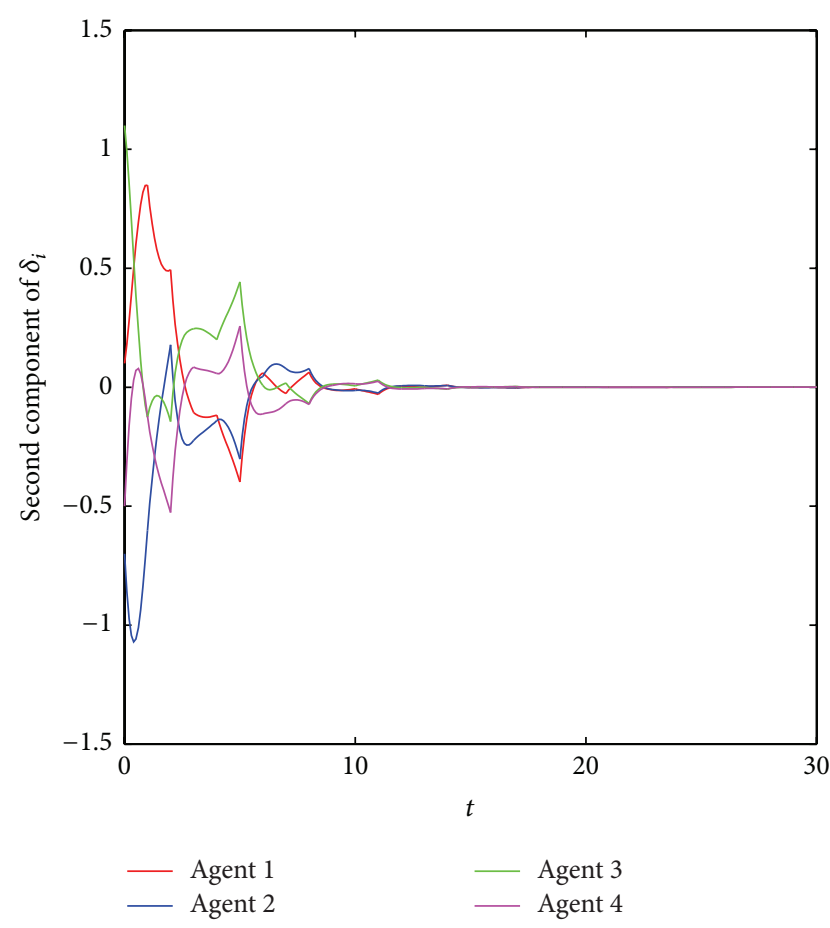

(b) $\tau(t)=0.1 \cos ^{2}(t)$

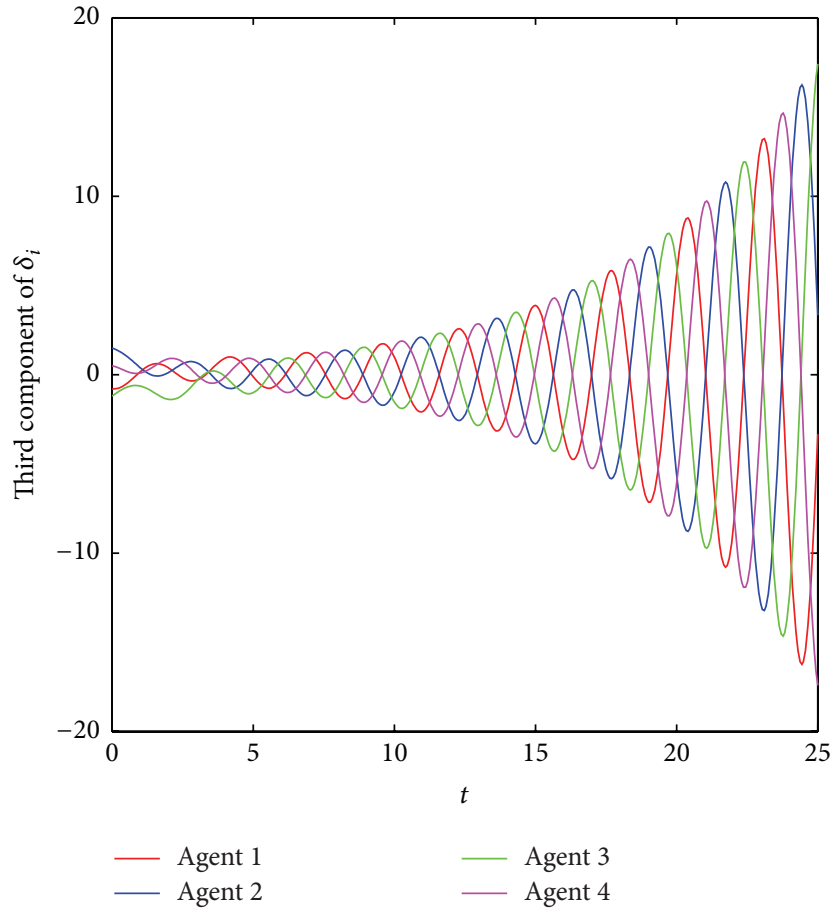

(d) $\tau(t)=0.3 \cos ^{2}(t)$

Figure 4: Consensus errors of multiagent system (2) with respect to different time-delay $\tau(t)$.

$p<(\ln N) / N$ (here, about $\left.6.9 \times 10^{-3}\right)$ in the large $N$ limit. Figure 5 reveals that consensus can still be achieved with a much smaller $p$ than the connectivity threshold indicating that the sufficient condition regarding connectivity in Theorem 8 can be weakened.

\section{Conclusion}

This paper has studied the continuous-time consensus problem of linear multiagent systems under Markovian switching interaction topologies, random noises, and time-varying 


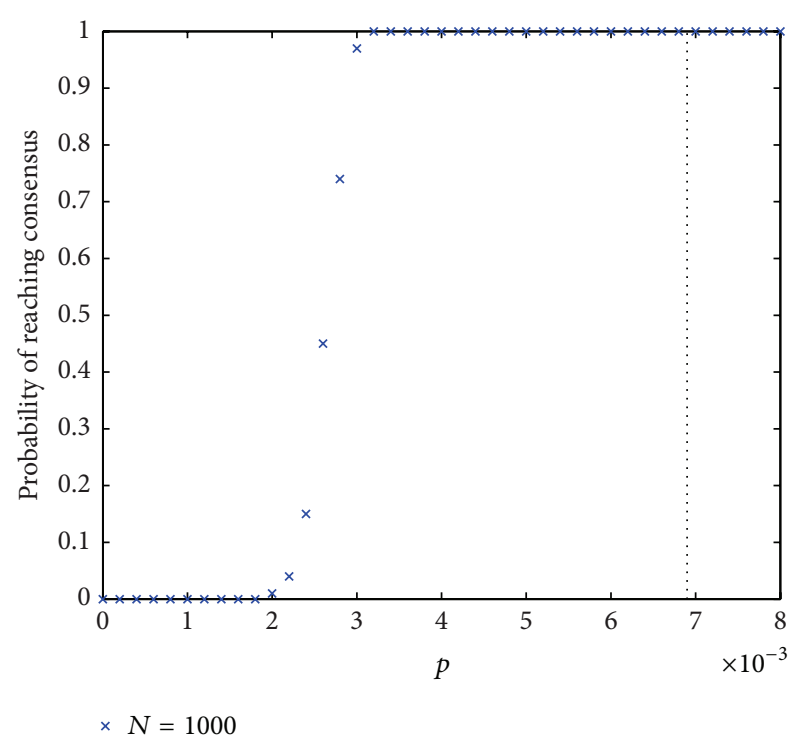

Figure 5: Probability of reaching consensus for multiagent system (1) as a function of $p$ over Markovian switching random graphs $\mathscr{G}(N, p)$.

delays. The agent dynamics are described by linear timeinvariant systems, in which two types of network-induced delays are considered, namely, delays affecting only the output of the agents' neighbors and delays affecting both the agents' own output and the output of their neighbors. Necessary and sufficient consensus conditions have been derived, respectively, for these two classes of multiagent systems. The design of consensus gains has a computationally advantageous decoupling feature. Numerical examples are given to demonstrate the effectiveness of the proposed methods. Although there is a gap between necessary and sufficient conditions, the necessary one seems to be tighter in view of the simulations. Future research worth investigation could be the heterogeneous time delays, general uncertainties, and systems with different dynamics (see, e.g., [53]).

\section{Conflict of Interests}

The author declares that there is no conflict of interests regarding the publication of this paper.

\section{Acknowledgments}

The author would like to thank Professor Quanxin Zhu for useful suggestions. The author is also grateful to the four anonymous reviewers and the editor for valuable comments that have improved the paper. This work was funded in part by the Program for Young Excellent Talents in Tongji University (2014KJ036) and by Shanghai Pujiang Program (15PJ1408300).

\section{References}

[1] J. N. Tsitsiklis, Problems in decentralized decision making and computation [Ph.D. thesis], Massachusetts Institute of Technology, Cambridge, Mass, USA, 1984.
[2] S. Chatterjee and E. Seneta, "Towards consensus: some convergence theorems on repeated averaging," Journal of Applied Probability, vol. 14, no. 1, pp. 89-97, 1977.

[3] R. Olfati-Saber and R. M. Murray, "Consensus problems in networks of agents with switching topology and time-delays," IEEE Transactions on Automatic Control, vol. 49, no. 9, pp. 15201533, 2004.

[4] A. Jadbabaie, J. Lin, and A. S. Morse, "Coordination of groups of mobile autonomous agents using nearest neighbor rules," IEEE Transactions on Automatic Control, vol. 48, no. 6, pp. 988-1001, 2003.

[5] R. Olfati-Saber, J. A. Fax, and R. M. Murray, "Consensus and cooperation in networked multi-agent systems," Proceedings of the IEEE, vol. 95, no. 1, pp. 215-233, 2007.

[6] W. Ren and R. W. Beard, "Consensus seeking in multiagent systems under dynamically changing interaction topologies," IEEE Transactions on Automatic Control, vol. 50, no. 5, pp. 655661, 2005.

[7] Y. Hong, G. Chen, and L. Bushnell, "Distributed observers design for leader-following control of multi-agent networks," Automatica, vol. 44, no. 3, pp. 846-850, 2008.

[8] W. Ren, "On consensus algorithms for double-integrator dynamics," IEEE Transactions on Automatic Control, vol. 53, no. 6, pp. 1503-1509, 2008.

[9] G. Xie and L. Wang, "Consensus control for a class of networks of dynamic agents," International Journal of Robust and Nonlinear Control, vol. 17, no. 10-11, pp. 941-959, 2007.

[10] K. You, Z. Li, and L. Xie, "Consensus condition for linear multiagent systems over randomly switching topologies," Automatica, vol. 49, no. 10, pp. 3125-3132, 2013.

[11] C.-Q. Ma and J.-F. Zhang, "Necessary and sufficient conditions for consensusability of linear multi-agent systems," IEEE Transactions on Automatic Control, vol. 55, no. 5, pp. 1263-1268, 2010.

[12] K. You and L. Xie, "Network topology and communication data rate for consensusability of discrete-time multi-agent systems," IEEE Transactions on Automatic Control, vol. 56, no. 10, pp. 2262-2275, 2011.

[13] Z. Li, Z. Duan, and G. Chen, "Dynamic consensus of linear multi-agent systems," IET Control Theory \& Applications, vol. 5, no. 1, pp. 19-28, 2011.

[14] Y. Shang, "Group pinning consensus under fixed and randomly switching topologies with acyclic partition," Networks and Heterogeneous Media, vol. 9, no. 3, pp. 553-573, 2014.

[15] Y. Hatano and M. Mesbahi, "Agreement over random networks," IEEE Transactions on Automatic Control, vol. 50, no. 11, pp. 1867-1872, 2005.

[16] M. Porfiri and D. J. Stilwell, "Consensus seeking over random weighted directed graphs," IEEE Transactions on Automatic Control, vol. 52, no. 9, pp. 1767-1773, 2007.

[17] A. Tahbaz-Salehi and A. Jadbabaie, "Consensus over ergodic stationary graph processes," IEEE Transactions on Automatic Control, vol. 55, no. 1, pp. 225-230, 2010.

[18] T. Li and J.-F. Zhang, "Consensus conditions of multi-agent systems with time-varying topologies and stochastic communication noises," IEEE Transactions on Automatic Control, vol. 55, no. 9, pp. 2043-2057, 2010.

[19] B. Touri and A. Nedić, "On ergodicity, infinite flow, and consensus in random models," IEEE Transactions on Automatic Control, vol. 56, no. 7, pp. 1593-1605, 2011. 
[20] G. Miao, S. Xu, and Y. Zou, "Necessary and sufficient conditions for mean square consensus under Markov switching topologies," International Journal of Systems Science, vol. 44, no. 1, pp. 178-186, 2013.

[21] I. Matei, J. S. Baras, and C. Somarakis, "Convergence results for the linear consensus problem under Markovian random graphs," SIAM Journal on Control and Optimization, vol. 51, no. 2, pp. 1574-1591, 2013.

[22] J. K. Hale and S. M. Verduyn Lunel, Introduction to Functional Differential Equations, Springer, New York, NY, USA, 1993.

[23] U. Münz, A. Papachristodoulou, and F. Allgöwer, "Delay robustness in consensus problems," Automatica, vol. 46, no. 8, pp. 1252-1265, 2010.

[24] U. Münz, A. Papachristodoulou, and F. Allgöwer, "Delay robustness in non-identical multi-agent systems," IEEE Transactions on Automatic Control, vol. 57, no. 6, pp. 1597-1603, 2012.

[25] X. Wang, A. Saberi, A. A. Stoorvogel, H. F. Grip, and T. Yang, "Consensus in the network with uniform constant communication delay," Automatica, vol. 49, no. 8, pp. 2461-2467, 2013.

[26] J. Liu, X. Liu, W.-C. Xie, and H. Zhang, "Stochastic consensus seeking with communication delays," Automatica, vol. 47, no. 12, pp. 2689-2696, 2011.

[27] Y.-Z. Sun and J. Ruan, "Leader-follower consensus problems of multi-agent systems with noise perturbation and time delays," Chinese Physics Letters, vol. 25, no. 9, pp. 3493-3495, 2008.

[28] Y. Shang, "Group consensus of multi-agent systems in directed networks with noises and time delays," International Journal of Systems Science, vol. 46, no. 14, pp. 2481-2492, 2015.

[29] J. Lai, S. Chen, and X. Lu, “Tracking consensus of nonlinear MASs with asymmetric communication delays in noisy environments," Communications in Nonlinear Science and Numerical Simulation, vol. 19, no. 7, pp. 2334-2344, 2014.

[30] Y. Shang, "Synchronization in networks of coupled harmonic oscillators with stochastic perturbation and time delays," Annals of the Academy of Romanian Scientists-Series on Mathematics and its Applications, vol. 4, no. 1, pp. 44-58, 2012.

[31] Y. Shang and R. Bouffanais, "Influence of the number of topologically interacting neighbors on swarm dynamics," Scientific Reports, vol. 4, article 4184, 2014.

[32] M. Cao, A. S. Morse, and B. D. O. Anderson, "Reaching a consensus in a dynamically changing environment: convergence rates, measurement delays, and asynchronous events," SIAM Journal on Control and Optimization, vol. 47, no. 2, pp. 601-623, 2008.

[33] N. Chopra, D. M. Stipanović, and M. W. Spong, "On synchronization and collision avoidance for mechanical systems," in Proceedings of the American Control Conference (ACC '08), pp. 3713-3718, Seattle, Wash, USA, June 2008.

[34] A. Papachristodoulou and A. Jadbabaie, "Synchonization in oscillator networks with heterogeneous delays, switching topologies and nonlinear dynamics," in Proceedings of the 45th IEEE Conference on Decision and Control, pp. 4307-4312, December 2006.

[35] L. Moreau, "Stability of multiagent systems with time-dependent communication links," IEEE Transactions on Automatic Control, vol. 50, no. 2, pp. 169-182, 2005.

[36] Y. Shang, "Average consensus in multi-agent systems with uncertain topologies and multiple time-varying delays," Linear Algebra and its Applications, vol. 459, pp. 411-429, 2014.
[37] Y. G. Sun, L. Wang, and G. Xie, "Average consensus in networks of dynamic agents with switching topologies and multiple timevarying delays," Systems \& Control Letters, vol. 57, no. 2, pp. 175183, 2008.

[38] T. Kailath, Linear Systems, Prentice-Hall, Englewood Cliffs, NJ, USA, 1980.

[39] O. L. V. Costa, M. D. Fragoso, and R. P. Marques, Discrete-Time Markov Jump Linear Systems, Springer, London, UK, 2005.

[40] X. Feng and K. A. Loparo, "Stability of linear Markovian jump systems," in Proceedings of the 29th IEEE Conference on Decision and Control, pp. 1408-1413, Honolulu, Hawaii, USA, December 1990.

[41] E. Seneta, Non-Negative Matrices and Markov Chains, Springer, New York, NY, USA, 2006.

[42] X. Mao, "Robustness of stability of stochastic differential delay equations with Markovian switching," Stability and Control: Theory and Applications, vol. 3, no. 1, pp. 48-61, 2000.

[43] Z. Zeng, J. Wang, and X. Liao, "Global asymptotic stability and global exponential stability of neural networks with unbounded time-varying delays," IEEE Transactions on Circuits and Systems II: Express Briefs, vol. 52, no. 3, pp. 168-173, 2005.

[44] Q. Zhu, J. Cao, and R. Rakkiyappan, "Exponential input-to-state stability of stochastic Cohen-Grossberg neural networks with mixed delays," Nonlinear Dynamics, vol. 79, no. 2, pp. 1085-1098, 2015.

[45] Q. Zhu and J. Cao, "Mean-square exponential input-to-state stability of stochastic delayed neural networks," Neurocomputing, vol. 131, pp. 157-163, 2014.

[46] Q. Zhu and J. Cao, "Stability analysis of markovian jump stochastic BAM neural networks with impulse control and mixed time delays," IEEE Transactions on Neural Networks and Learning Systems, vol. 23, no. 3, pp. 467-479, 2012.

[47] X. Mao, A. Matasov, and A. B. Piunovskiy, "Stochastic differential delay equations with Markovian switching," Bernoulli, vol. 5, pp. 1-18, 1999.

[48] J. S. Caughman and J. J. P. Veerman, "Kernels of directed graph Laplacians," Electronic Journal of Combinatorics, vol. 13, article R39, 2006.

[49] S. Boyd, L. E. Ghaoui, E. Feron, and V. Balakrishnan, Linear Matrix Inequalities in System and Control Theory, Society for Industrial and Applied Mathematics, Philadelphia, Pa, USA, 1994.

[50] H. Weyl, "Das asymptotische Verteilungsgesetz der Eigenwerte linearer partieller Differentialgleichungen (mit einer Anwendung auf die Theorie der Hohlraumstrahlung)," Mathematische Annalen, vol. 71, no. 4, pp. 441-479, 1912.

[51] J. G. VanAntwerp and R. D. Braatz, "Tutorial on linear and bilinear matrix inequalities," Journal of Process Control, vol. 10, no. 4, pp. 363-385, 2000.

[52] B. Bollobás, Random Graphs, Cambridge University Press, New York, NY, USA, 2001.

[53] Y. Zheng and L. Wang, "Consensus of heterogeneous multiagent systems without velocity measurements," International Journal of Control, vol. 85, no. 7, pp. 906-914, 2012. 


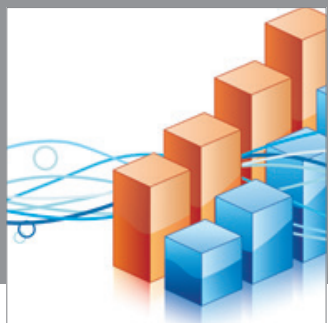

Advances in

Operations Research

mansans

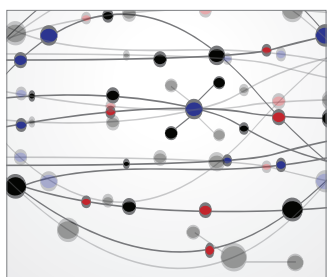

The Scientific World Journal
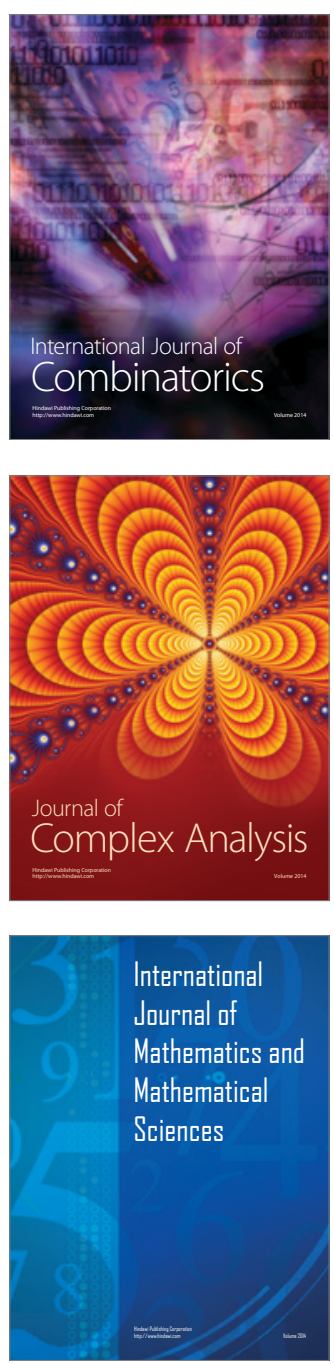
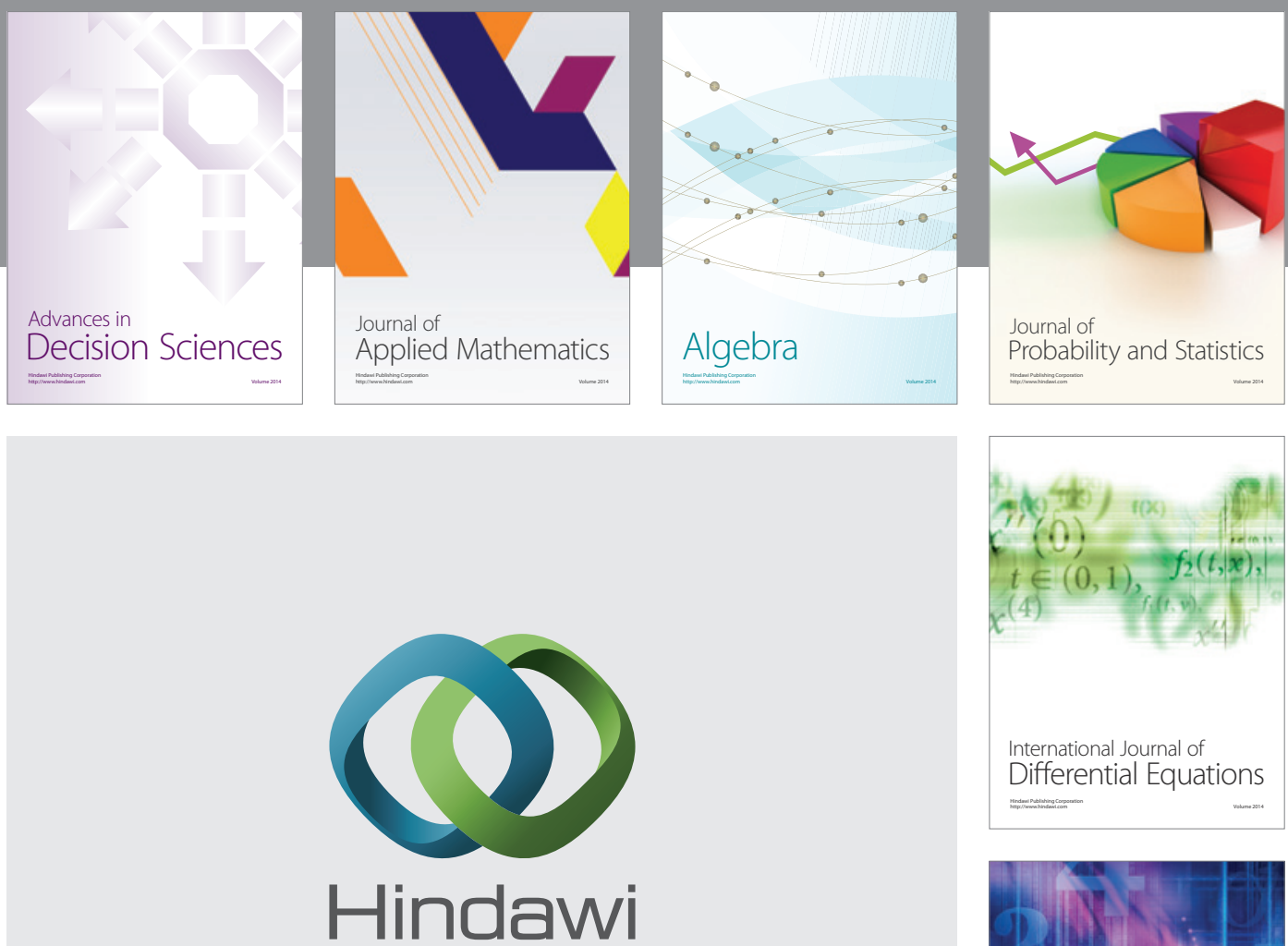

Submit your manuscripts at http://www.hindawi.com
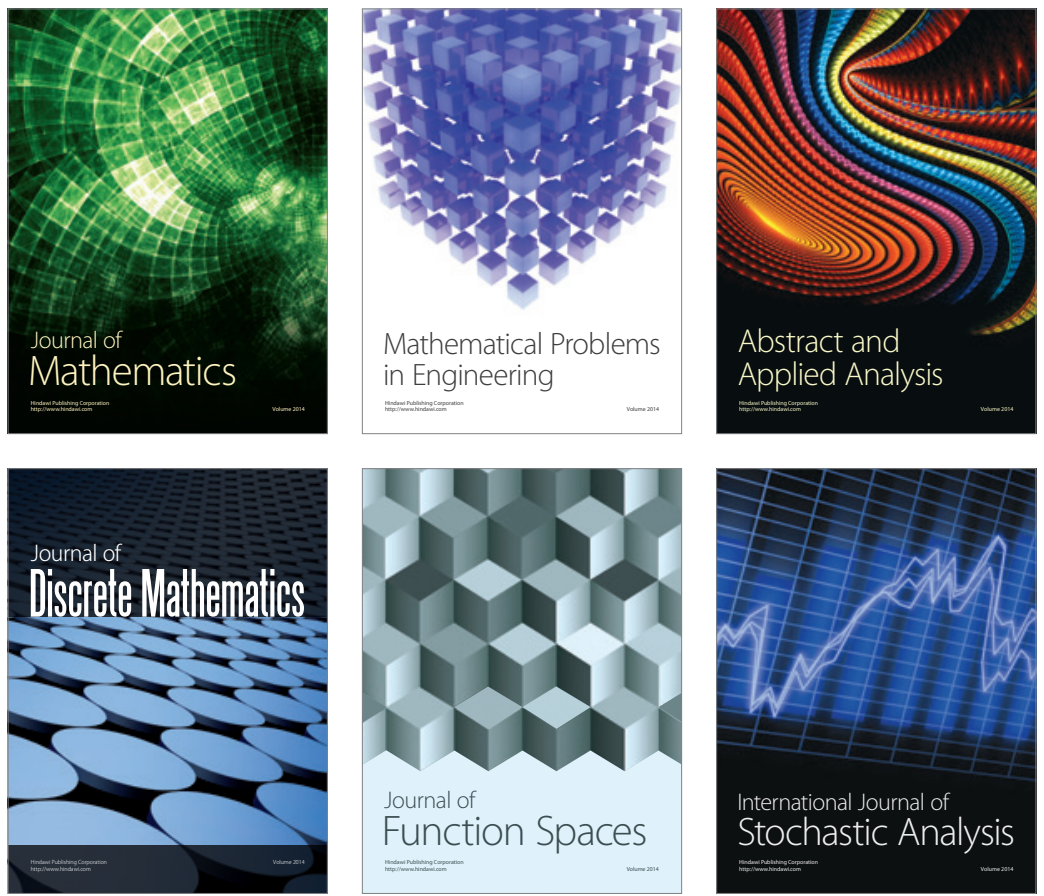

Journal of

Function Spaces

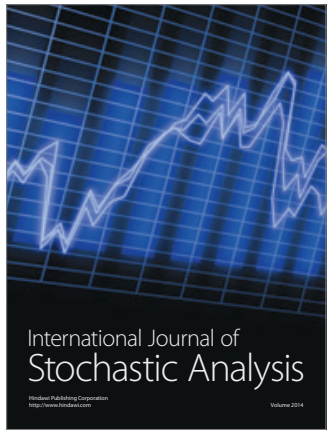

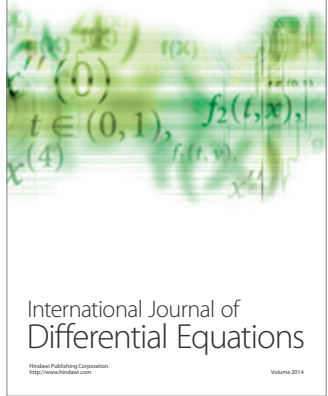
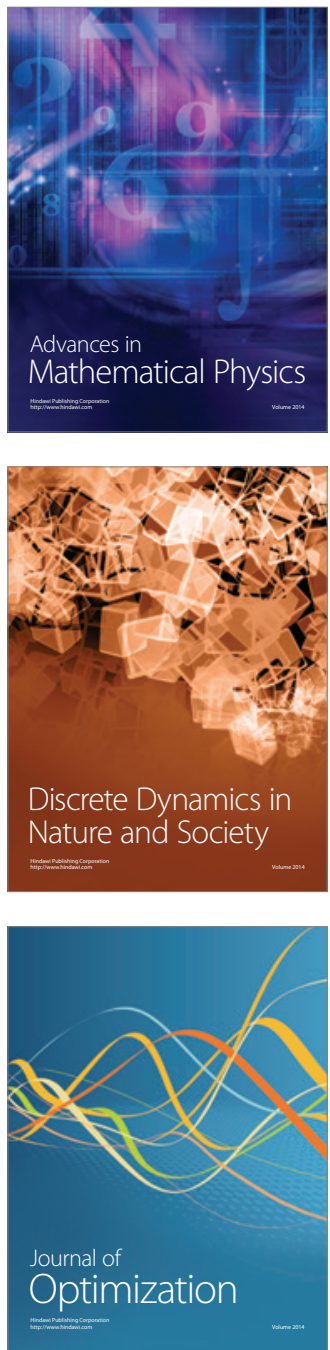